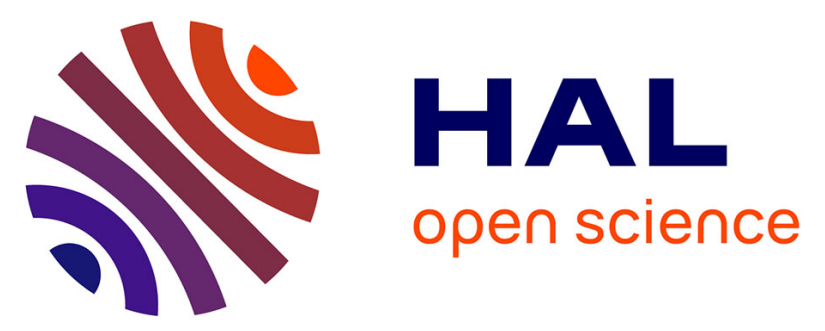

\title{
Variations on the same BANC Category 8 theme: Towards the Development of a High Fidelity Acoustic Hybrid Method using Computational AeroAcoustics
}

S. Redonnet, G. Cunha

\section{- To cite this version:}

S. Redonnet, G. Cunha. Variations on the same BANC Category 8 theme: Towards the Development of a High Fidelity Acoustic Hybrid Method using Computational AeroAcoustics. AIAA AVIATION 2014, Jun 2014, ATLANTA, United States. hal-01069401

\section{HAL Id: hal-01069401 \\ https: / hal-onera.archives-ouvertes.fr/hal-01069401}

Submitted on 29 Sep 2014

HAL is a multi-disciplinary open access archive for the deposit and dissemination of scientific research documents, whether they are published or not. The documents may come from teaching and research institutions in France or abroad, or from public or private research centers.
L'archive ouverte pluridisciplinaire HAL, est destinée au dépôt et à la diffusion de documents scientifiques de niveau recherche, publiés ou non, émanant des établissements d'enseignement et de recherche français ou étrangers, des laboratoires publics ou privés. 


\title{
Variations on the same BANC Category 8 theme: Towards the Development of a High Fidelity Acoustic Hybrid Method using Computational AeroAcoustics
}

\author{
Stéphane Redonnet*, Guilherme Cunha ${ }^{\dagger}$ \\ ONERA (French Aerospace Centre), BP 72 - 29 av Division Leclerc, Châtillon, 92322, France
}

\begin{abstract}
In regard to the mitigation of noise annoyance by aircraft, the present work addresses acoustic hybrid approaches, which allow simulating a given noise problem via successive weakly-coupled computations. Within that context, over the past four years, several R\&D works were conducted at Onera so as to improve and optimize an already existing high fidelity acoustic hybrid approach, which noise propagation stage relies on a Computational Aeroacoustics (CAA) method. Consisting in dedicated theoretical studies, algorithmic developments and numerical simulations, these $R \& D$ works resulted in fundamental insights and methodological innovations. The latter were all validated against various test cases of increasing complexity, which were mostly derived from test cases proposed within the Benchmark for Airframe Noise Computations (BANC) initiative supported by NASA. This shows how international benchmark efforts such as the ones promoted through BANC initiative constitute a key means for improving numerical methods in acoustics, whether it is by offering to consolidate further already existing approaches, or by helping in making novel techniques emerge.
\end{abstract}

\section{Introduction}

few years ago, noise annoyances by aircraft were officially identified as the major obstacle to a sustainable growth of air traffic. Therefore, all stakeholders involved in the development of aircraft systems or components now focus on practical ways to reduce the acoustic signature by their products. On another hand, since acoustics is a complex discipline, they are often bounded to make an intensive use of numerical simulations, which constitutes a powerful R\&D tool, when used in complement to experimentation. This, however, requires a continuous development and a proper validation of the modeling and solving techniques that are to be used for simulating the noise generation and/or propagation phenomena occurring in realistic situations.

\section{Aircraft Noise Prediction via Acoustic Hybrid Methodologies}

\section{A. Aircraft Noise}

The noise signature of an aircraft includes two main contributions, respectively of propulsive and non propulsive origins. The first one, namely the engine noise, is due to all propulsive devices by the engines (turbojet or turboprop), whereas the second one, namely the airframe noise, is induced by the airframe and its appendices (fuselage, wings, slats, flaps, landing gears, cavities, etc.). Although the engine noise accounts for a dominant portion of the overall aircraft noise during take-off, the airframe noise component becomes equally important during the approach for landing, when the engine thrust is considerably reduced. From a more phenomenological point of view, such a distinction between engine and airframe noises vanishes at some point, since both components result from the contribution and combination of a large number of acoustic sources and phenomena. Indeed, noise finds its origin in numerous source mechanisms such as structural vibrations, fluidic motions, flow interactions with structures, gas combustion or explosions, and so on. Once they have been generated by these sources, acoustic waves propagate within the surrounding environment, which is generally constituted with one or several media of various complexity (e.g. comprising solid bodies and/or medium heterogeneities, etc.). During such propagation phase, acoustic waves may be submitted to numerous and important alterations in terms of amplitude, phase or frequency, which all result from mechanisms as diverse as reflection and diffraction effects by solid structures, convection by fluidic motions, refraction by the medium heterogeneities, diffusion by the medium turbulence, absorption by the medium viscosity, and so on.

\footnotetext{
* PhD, Research Scientist, CFD and Aeroacoustics Department, Onera, Stephane.Redonnet@onera.fr.

${ }^{\dagger}$ PhD, Research Scientist, CFD and Aeroacoustics Department, Onera, Guilherme.Cunha@onera.fr.
} 


\section{B. Hybrid Methods for the Aircraft Noise Prediction}

Many of the acoustic generation processes and most of the acoustic propagation mechanisms are relevant to the physics of fluid dynamics, and can thus be simulated by numerically solving the Navier-Stokes equations. At the present time, however, and despite the continuous development of computational tools and resources, it is still extremely challenging to solve aeroacoustic problems following a direct manner, that is to say, via a single calculation. Indeed, except in particular situations (e.g. academic configurations), it is nearly impossible to simulate at the same time the noise generation and its subsequent propagation, which underlying mechanisms greatly differ by their intrinsic characteristics (e.g. energy, length scales, etc.). As an example, most of the noise annoyances due to modern aircraft come from the so-called aerodynamic noise, which results from either the interaction of airflow with the structure itself (e.g. airframe noise), or from its ingestion by the engines (e.g. fan and/or turbine noises, etc.). On another hand, the aerodynamic noise physics is made of complex phenomena covering a broad range of spatio-temporal scales, with acoustic generation processes that are driven by turbulent structures of high amplitude and small space-time correlations, while propagation ones are associated with sound waves of low amplitudes and large space-time correlations. Thus, and although both phenomena are ruled by the same compressible Navier-Stokes equations, they cannot be easily predicted via a single calculation because the computational resources required to resolve all of the relevant scales would be far too high. Therefore, to make the numerical approach tractable in a practical context, the overall acoustic problem is usually decomposed into a set of coupled sub-problems that focus on individual sub-regions of the overall spatial domain. Each subproblem has a specific range of amplitudes and physical scales that can be addressed using a numerical method that is customized to the dominant physics occurring at this stage. Methods involving a mix of techniques in this manner are classified as hybrid approaches for the acoustic prediction.

\section{Multi-stage Coupling for Hybrid Methods in Aeroacoustics}

A critical aspect of developing acoustic hybrid methodologies corresponds to the coupling, i.e. the information exchange to occur between the various stages respectively associated with the individual sub-problems. The nature of this coupling is problem dependent, because of significant variations in the inter-dependencies between the various stages from one problem to another. However, except in problems involving acoustic feedback (e.g. screech tones, in jet aeroacoustics), the coupling between these stages is weak, i.e. primarily unidirectional. Under this scenario, feedback from a given stage to the previous one can be neglected, and the successive stages of an acoustic hybrid calculation can be coupled in a weak sense, all possible retro actions from a given step to the previous one being then neglected. Such a weak-coupling process to occur between two successive stages of a computational acoustic hybrid approach is constituted with a data transfer, which role is to transmit to the next level all the acoustic information gathered at each step. Needless to say, such an operation must be properly achieved, so that it does not degrade the acoustic signal information to be transmitted. This requires that the weak-coupling technique to be used both relies on sound physical principles and offers sufficient numerical robustness, especially in regard to an application within a realistic context.

\section{Noise Propagation Stage and Computational AeroAcoustics}

Regarding more specifically the noise propagation stage of an acoustic hybrid method, its role is to propagate within the surrounding environment all the acoustic information that could have been made available from any prior noise generation modeling and/or simulation. As said above, due to the variety and complexity of all physical phenomena involved, numerically simulating such a propagation phase is generally not trivial. In particular, computational techniques required for handling such noise propagation stage must simulate accurately the propagation of acoustic waves over relatively large distances across possibly heterogeneous media, while accounting for the possible presence of solid obstacles. This may typically be accomplished with higher fidelity acoustic propagation approaches relying on the Euler equations or a linearized version thereof, such as a Computational AeroAcoustics (CAA) method. Indeed, one can here recall that only a CAA method can simultaneously account for both the reflection/diffraction effects by solid obstacles and the refraction effects by the flow heterogeneities, in contrast to other techniques that can only model the former (such as the Boundary Element Method, BEM), or even neither of them (such as an Integration Method, IM, whether it is based on a Kirchhoff ${ }^{1}$ extrapolation or an Acoustic Analogy $\left.y^{2,3}\right)$. As an illustration of typical aircraft problems to be preferably solved via an acoustic hybrid method relying on a CAA-based propagation stage, one can exemplify those internal noise propagation problems that occur in nacelle and exhaust ducts of engines. Indeed, here, once their generation has been properly modeled by analytical means (e.g. duct mode theories ${ }^{4}$ ) or simulated via a numerical method (e.g. Computational Fluid Dynamics, CFD), acoustic waves may then be transferred to a CAA solver, for the latter to propagate them through the duct, while accounting for all internal effects to be possibly induced by the presence of flow heterogeneities, solid devices or any other disturbing elements (such as noise absorbing panels, etc.). Another typical situation where an acoustic hybrid approach based on a CAA propagation stage can be advantageously applied corresponds to these external noise problems which propagation phase occurs within a complex environment, such as for instance the airframe noise emission by 
aircraft appendices (e.g. landing gear, etc.). Indeed, here again, once their generation has been properly simulated (usually via an unsteady compressible CFD method), acoustic waves may then be transferred to a CAA solver, for the latter to propagate them up to the far-field, while accounting from all the installation effects induced by either the airframe elements (e.g. reflection/diffraction) or the airflow that surrounds the latter (e.g. convection/refraction).

\section{E. Weak-coupling Techniques for Computational AeroAcoustics}

As was said, properly weak-coupling a preliminary noise generation stage with a CAA-based propagation one implies that the acoustic information is transferred from the former to the latter in a conservative fashion, that is, without any loss nor duplication. This requires that the weakcoupling technique to be used relies on sound physical principles, as well as it offers sufficient numerical robustness for being applicable to realistic configurations. In particular, the technique must possess and maintain certain critical attributes; it must first offer enough generality with respect to the physics, being in particular free of any too stringent assumption that would restrict its application to only a certain type of situation (e.g., homogeneous

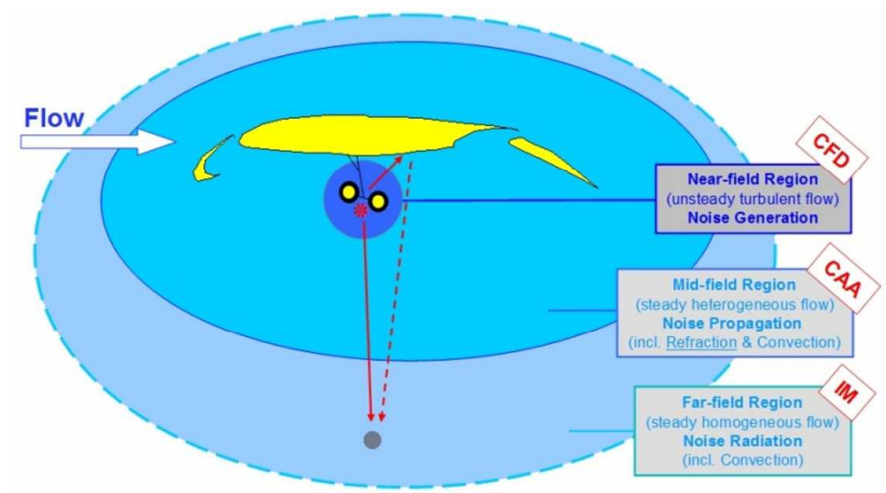

Figure 1 - Aircraft noise emission by aircraft undercarriage systems. Sketch of the noise emission by a landing gear installed under a high-lift wing, to be numerically predicted via a high fidelity acoustic hybrid approach based on weakly coupled calculations (CFD-CAA-IM).

medium, low Mach number flow, etc.). It must then guarantee enough consistency with respect to the modeling, being in particular free of any hidden bias coming from the mismatch that might arise between the respective continuous formulations underlying the noise generation and the CAA-based propagation stages (e.g. viscous effects, nonlinear effects, etc.). It must also offer sufficient stability with respect to the numerical solving, being in particular free of any issues that might arise from the mismatch between the respective discrete formulations associated with these two latter stages (e.g. interpolation effects, under-solving discretization effects, etc.). Last but not least, for being applicable to many situations of interest, the coupling technique must offer a perfect numerical anechoicity (or transparency), that is, must allow handling properly the acoustic backscatter effects that may occur within the noise propagation regions. Indeed, regarding for instance internal propagation problems, one can here recall how sound waves that propagate within a duct can be more or less importantly backscattered because of various artifacts such as those constituted by either the termination or any additional devices (bifurcations, splices, etc.) of the duct, as well as by the lining of the latter with noise absorbing materials. On another hand, one can also exemplify external propagation problems involving installed configurations, where any noise source region is likely to be surrounded by solid bodies that may backscatter anything it radiates (consider, for instance, the aerodynamic noise emission by a landing gear to be installed under a wing - see Fig. 1). Needless to say, for the overall hybrid calculation to behave correctly in these situations, not only the coupling technique must transfer accurately the acoustic information from the prior noise generation stage to the CAA-based propagation one, but it must also allow any of the acoustic occurrences that may be backscattered by the latter onto the coupling area to cross it following a non reflective (or anechoic) manner, that is, without being bounced back or without creating spurious numerical noise.

\section{Onera's Past Efforts and Recent Advances on the Development of a CAA-based Aeroacoustic Hybrid Method}

\section{A. Past Efforts}

Since more than a decade, Onera largely promoted the multi-stage aeroacoustic hybrid approach, by both i)

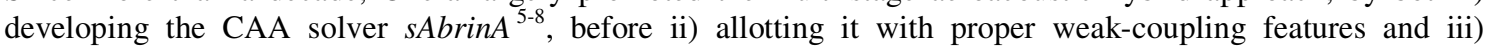
applying it to various aircraft noise problems. In particular, with the view of deriving a general weak-coupling technique that could allow transferring properly any given acoustic signal coming from a prior noise generation stage to within a CAA-based propagation one, the present first author had initially developed an original interfacing technique ${ }^{5,6}$. The latter technique consisted in forcing explicitly the CAA computed field with the acoustic signal to be transferred, such explicit forcing being applied at each time step and over a few rows of ghost cells (see details in Refs. 5, 6). As is, such interfacing technique was not only straightforward to implement and use, but it was also general enough for being applicable to many situations. Regarding internal noise propagation problems for instance, and as left side of Fig. 2 shows, such interfacing technique was successfully 
applied to various cases (e.g. the aft fan noise emission by turbojet engines ${ }^{7,8}$ ), for which the acoustic signals to be CAA-forced were derived via analytical means (based on the modal theory ${ }^{4}$ ). On another hand, regarding now external noise problems, and as exemplified in right side of Fig. 2, the technique was also applied with success to several airframe noise applications (e.g. the acoustic emission by either an in-flight NACA0012 airfoil with a blunted trailing edge $\mathrm{e}^{9,10}$ or a thick plate embedded within a flow ${ }^{11}$ ), the acoustic signals to be CAA-forced being, this time, obtained via a compressible unsteady CFD calculation.

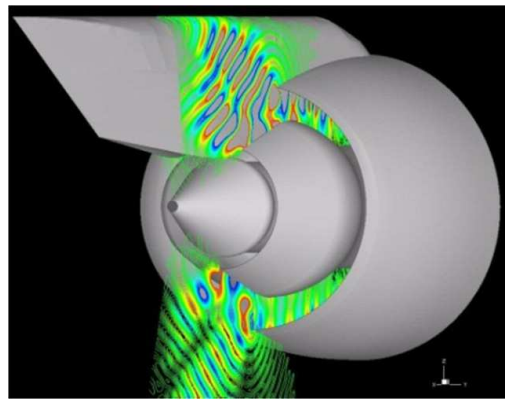

Figure 2. Aircraft noise prediction using an acoustic hybrid method based on a CAA noise propagation stage. Top: aft fan noise emission by a realistic exhaust, via a hybrid calculation based on both an analytical noise generation and a CAA noise propagation stages. Right side; trailing edge noise emission by a blunted airfoil, via a hybrid computation relying on an unsteady CFD noise generation and a CAA (resp. IM) noise propagation (resp. radiation) stages.

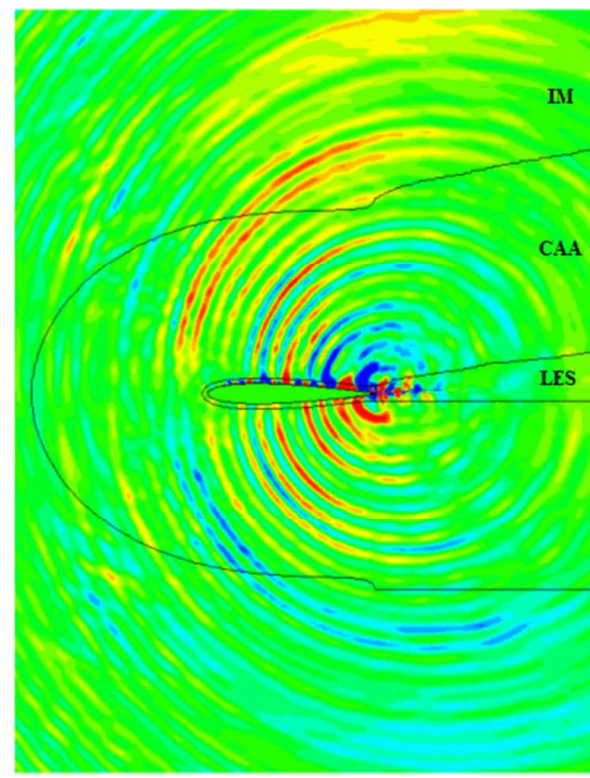

\section{B. Recent Advances, with Validation Efforts based on BANC Benchmark Exercises}

At that stage however, it was needed to improve further and, then, and to optimize the overall CAA-based hybrid approach, so that it becomes accurate and robust enough for handling properly complex aircraft noise problems. More precisely, it was first required to improve the interfacing technique itself so that it can handle acoustic backscattering phenomena, for the resulting weak-coupling procedure become applicable to many situations of interest (see the discussion above). Then, it was needed to improve the ways the latter weak-coupling procedure can cope with all stringent constraints that are dictated by real-life applications, without being jeopardized by some of their unavoidable side effects (such as the signal degradation to which unsteady CFD data may be subjected, when manipulated for being acoustically exploited, see detail below). With the view of fulfilling those two needs, several R\&D actions were conducted; these works, which consisted in the development of innovative solutions for an optimization of the CAA-based hybrid approach, constitute the matter of the present article. Please, note that whereas some of these works had been already documented (more or less extensively) through previous communications at AIAA Conferences ${ }^{12-16}$, others constitute fresh material. The reason for gathering altogether these various insights within the same present article was twofold; first, all these R\&D actions targeted a common objective, which is the optimization of a CAA-based aeroacoustic hybrid method. Second, and as the present paper clearly shows, these actions benefited directly from the so-called BANC (Benchmark of Airframe Noise Computations) initiative proposed by NASA $/ \mathrm{LaRC}^{17}$; indeed, in order to both i) validate accurately the developments achieved and ii) illustrate their potentialities with respect to realistic aircraft noise problems, various test cases derived from the BANC Category 8 benchmark exercises were here addressed. This shows how international benchmark efforts such as the ones promoted through the BANC initiative constitute a key means for improving numerical methods in acoustics, whether it is by i) offering to consolidate further already existing approaches, or by ii) helping in making novel techniques emerge.

\section{Some Details about the BANC-Based Validation Efforts}

In a first time, validation efforts focused on a rather simple hybrid scenario, which consisted in weak-coupling both an analytical noise generation stage and a CAA-based noise propagation one. For doing so, only academic test cases were considered, among which the noise scattering problem proposed in BANC Category 8.1 (see sections IV.B and V.B, below). For each case, the hybrid calculation consisted in forcing a CAA computation with an acoustic signal of analytical nature, such signal being CAA-forced at each time step along an immaterial interface delimiting a given source region. Please, note that, thanks to its analytical nature, the acoustic source signal could be defined exactly where and when needed by the CAA stage, that is, at the exact space-time locations required by the CAA-forcing. Such signal escaped thus the need to be submitted to any space and/or time interpolation / sampling operations, which could ever have degraded its accuracy due to spuriousing and aliasing phenomena, questioning thus the present validation effort. Regarding these analytically-based hybrid calculations, all CAA computations were performed with the help of ONERA's sAbrinA solver ${ }^{5-8}$ (Redonnet et al, 2001-2013), being conducted over one core of a laptop (64 bits, Pentium 2.1GHz, 4Gb Ram) and requiring 
approximately 30 minutes of wall clock time. One can here recall that $s A b r i n A$ is a structured, time-accurate CAA code that solves either the full or the linear Euler equations, in a conservative and perturbed form (with a splitting of the complete variables into a 'frozen' mean flow and a 'fluctuating' perturbation). The solver employs high-order, finite-difference (FD) operators, involving high-order spatial derivatives and filters, as well as a $3^{\text {rd }}$ order, multi-stage, Runge-Kutta time-marching scheme. The code deals with multi-block structured grids with one-to-one interfaces, and is fully parallelized using the Message Passing Interface (MPI) standard. Finally, the solver includes the usual boundary conditions (reflection by solid walls, non-reflecting and free-field radiation ${ }^{5,6}$, etc.), as well some unique to specific applications (such as the Non Reflective Interface technique ${ }^{12}$ that was extensively used in the present effort). More detailed information about the sAbrinA solver and its underlying methodology can be found in Refs. 5-6.

In a second time, with the view of assessing further the optimized aeroacoustic hybrid approach as well as illustrating better the potentialities offered by the latter with respect to an application to realistic noise problems, validation works focused on a more complex hybrid scenario, that is, relying on a noise generation stage based on compressible unsteady CFD. To that end, several airframe noise problems of increasing complexity were considered, being all solved following a hybrid process relying on weakly coupled CFD and CAA calculations. Therefore, for each problem, a preliminary unsteady compressible CFD calculation was first acquired, from which a proper acoustic source signal was then derived, before a subsequent CAA calculation was forced with it. The two major problems addressed here were directly derived from the benchmark exercises proposed in Category 8 of BANC-II and BANC-III, respectively (noise radiation by a Tandem Cylinder and by a simplified Nose Landing Gear, respectively - see section IV.C and VI, respectively). For these various CFD-CAA hybrid calculations could effectively be achieved, several preliminary (though sometimes intensive) tasks were first required, such as i) the proper specification of the CFD storage to be achieved in space and/or time, ii) the analysis of the CFD stored signal, iii) the derivation of a suitable CAA set-up (in terms of space/time grids, steady mean flow, etc.), and iv) the proper derivation of the source signal to be CAA-forced. Regarding that last stage, and unlike what had been done for the previous analytically-based hybrid calculations (where it was analytically derived according to the CAA set-up), each source signal had to be sampled and/or interpolated in space and/or time, so that it matches the CAA discretization requirements. This, however, was achieved with a special attention paid on the signal degradation to be possibly induced by aliasing and/or spuriousing effects, which were here minimized at best thanks to the use of the innovative techniques (dedicated analysis and interpolation tools) developed in the present framework (see section V.A below). As for the previous analytically-based hybrid calculations, the CAA stage was handled with the help of $s A b r i n A$ solver. However, due to the more important requirements in terms of computational resources (grid size, time duration, etc.), these CAA calculations were run in parallel, on various machines. In particular, the CAA calculations associated with the test case derived from BANC-II Category 8 (Tandem Cylinder, see section IV.C below) were run over 64 cores of a NASA/LaRC cluster*, requiring up to $18 \mathrm{~h}$ of cumulated CPU (for a total of 12,660 iterations and 850,000 grid points computed). On another hand, the CAA calculations associated with the case from BANC-III Category 8 (Nose Landing Gear, see section VI below) were run over 480 cores of Onera super computer ${ }^{\dagger}$, requiring approx. $45 \mathrm{CPU}$ hours each (for a total of 12,000 iterations and 66 millions of grid points computed).

\section{Development of the Non Reflective Interface, and Validation/Application through Test Cases from BANC Category 8}

\section{A. Development of the Non Reflective Interface (NRI)}

First action consisted in enabling the interfacing technique to deal with possible back scattering of acoustic waves through the weak-coupling interface - a thing likely to occur whenever installed configurations are addressed, since solid bodies surrounding the source region can reflect back anything the latter radiates. Indeed, in its original version, ${ }^{5,6}$, and because of the explicit forcing it relied on, the interfacing technique was acting as a potentially reflective (or solid wall) boundary condition, sending back within the CAA domain any acoustic occurrence it could be impinged by. Although such a potentially reflective character of the interface was not really an issue for isolated configurations (such as the trailing edge noise one previously recalled), it could constitute a severe drawback - not to say a contraindication - for installed configurations, where solid bodies may surround the coupling region, and then backscatter anything the latter radiates. As an example, let us consider the noise emission by a landing gear, to be installed under a wing (see Fig. 3); in such a situation, although it could effectively allow transferring the acoustic information from a prior noise generation to the CAA-based propagation one, due to its non-anechoic character, the original interfacing technique was not able handling properly the acoustic occurrences to be possibly backscattered onto the weak-coupling area, because of

\footnotetext{
*Intel P4 $2.53 \mathrm{GHz}$ PC cluster with Myrinet network.

${ }^{\dagger} 160$ Westmere bi-processors, with each processor composed of 6-cores X5675 of 3,07 GHz/ $4 \mathrm{Go}$.
} 
the wing. With the view of removing such a limitation, the interfacing technique was thus entirely refurbished, so that it is allotted a non reflective character. Such improved version of the technique, which is called hereafter Non Reflective Interface (NRI) ${ }^{12}$, relies on a composite representation of the CAA mean and perturbed fields,

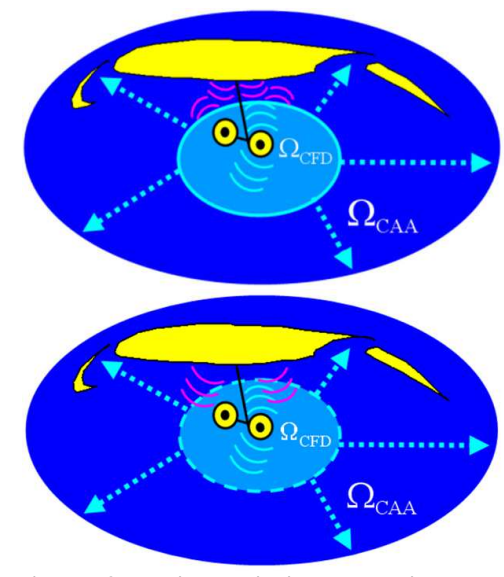

Figure 3. Noise emission by an installed landing gear noise, as predicted via a hybrid approach based on CFD-CAA weakly coupled calculations. Reflective (top) and non-reflective (NRI, bottom) $C F D \rightarrow C A A$ interfacing techniques. which results from a dual (rather than the sole classical) definition of the flow splitting (see detail in Ref. 12). Such a dual splitting allows prescribing differently both fields within a same CAA domain, depending on where the computed point is located. Thanks to their discontinuous nature, these mean/perturbed fields can be allotted a given discontinuity that, occurring along the coupling interface, corresponds specifically to the source signal to be transferred from the noise generation regions to the acoustic propagation ones* Thanks to such NRI technique, the source signal can thus be accurately transferred within the CAA calculation domain, passing from the noise production zones (over which it is left CAAuntouched) to the propagation areas (over which it becomes free of CAA-evolving). More important, the CAA perturbed field being not submitted to any other treatment than such an implicit forcing by the source signal, any other disturbance can travel within the whole domain, independently of where the NRI weak-coupling interface is located. Therefore, the CAA perturbed field remains free of evolving as it has to do, and in particular of handling backscattered occurrences that may arise within the noise propagation regions - a thing that, again, is mandatory for the resulting NRI-based weakcoupling procedure and CAA-based hybrid approach are applicable to realistic problems.

\section{B. Validation of NRI through BANC Category 8.1}

As shown below, the NRI technique was first validated via the academic test case excerpted from the BANC Category 8.1, which addresses the acoustic scattering by a $3 \mathrm{D}$ sphere of a quadrupole source within an infinite quiescent medium. Here, it can be recalled that such test case was jointly designed by NASA, JAXA and Onera, so as to serve as a generic problem for assessing the various ways of far-field extrapolating a set of near-field unsteady data. Indeed, for this problem, there exists an analytical solution ${ }^{\dagger}$ from which one can feed any acoustic extrapolation tool (in the exact same manner of what would be done with unsteady perturbations coming from a prior CFD calculation, for less academic situations).

We thus consider here a sphere of radius $R$ located at the origin $\mathbf{x}=\mathbf{0}$ of a quiescent medium. Such sphere acts as a scattering agent onto a quadrupolar acoustic source that is located $1.5 R$ away from the sphere's center (at $\mathbf{x}=(-1.5 R ; 0 ; 0)$ ) and that pulsates with a reduced frequency $k R=2 \pi R$. Following guidelines of BANC benchmark, the near-field region was chosen as the area encompassing both the source and the sphere $\left(\Omega_{\mathrm{S}}=[-\right.$ $2 R ; 1.5 R] \times[-1.5 R ; 1.5 R] \times[-1.5 R ; 1.5 R])$. From such source region $\Omega_{\mathrm{s}}$, the unsteady signal resulting from both the acoustic emission by the quadrupole and its scattering by the sphere was NRI-forced within a wider CAA calculation domain $\Omega_{\mathrm{CAA}}=[-5 R ; 5 R]^{3}$. Such NRI forcing of the CAA perturbed field with the analytical source signal was achieved every iteration, being applied along an immaterial interface given by the cubic envelop corresponding to the frontiers of the source region $\Omega_{\mathrm{s}}$ (blue dashes in Fig. 4). At this stage, one can precise that, for the CAA stage consumption, and although this could have been done, the sphere obstacle was not meshed, for the propagation grid can be taken as an ideal one, that is, of a homogeneous Cartesian type. The reason for doing so was to minimize at best all possible side-effects (such as metric errors) that could have biased the validation exercise. Such a Cartesian CAA grid was allotted a space discretization corresponding to $\Delta_{\mathrm{x}}=\Delta_{\mathrm{y}}=\Delta_{\mathrm{z}}$ $=R / 10$, leading to a computational domain comprised of $101^{3}$ (i.e. approx. 1 million) points. Such a grid ensured a nominal value of $11 \mathrm{PPW}$ (Points Per Wavelength), guaranteeing thus a maximal accuracy error of $0.1 \%$ in terms of acoustic group velocity (all this, with respect to the 7 point $/ 6^{\text {th }}$ order finite differences schemes used by the CAA solver). The computation was conducted for a simulation time corresponding to 6 source periods, with 20 iterations per period, leading to a CFL (Courant-Friedrichs-Lewy) number of 1/2. Figure 4 displays the instantaneous perturbed pressure field obtained at the end of the $6^{\text {th }}$ source cycle, either via the NRI-forced CAA calculation, or via the analytical evaluation; as one can see, both results collapse all over the computational box, included close to the NRI interface (depicted in blue dashes). This proves that the source signal was accurately

\footnotetext{
${ }^{*}$ When the CAA method relies on finite-differences (FD) schemes, a local correction of the space (derivation and filtering) operations allows handling properly such discontinuity of the mean/perturbed fields, which sum (i.e. total field) remains however perfectly continuous over the whole domain

${ }^{\dagger}$ which was here provided by Dr. Ikeda, from JAXA.
} 
NRI-forced and then CAA-propagated within the calculation domain, which further validates the Non Reflective Interface.
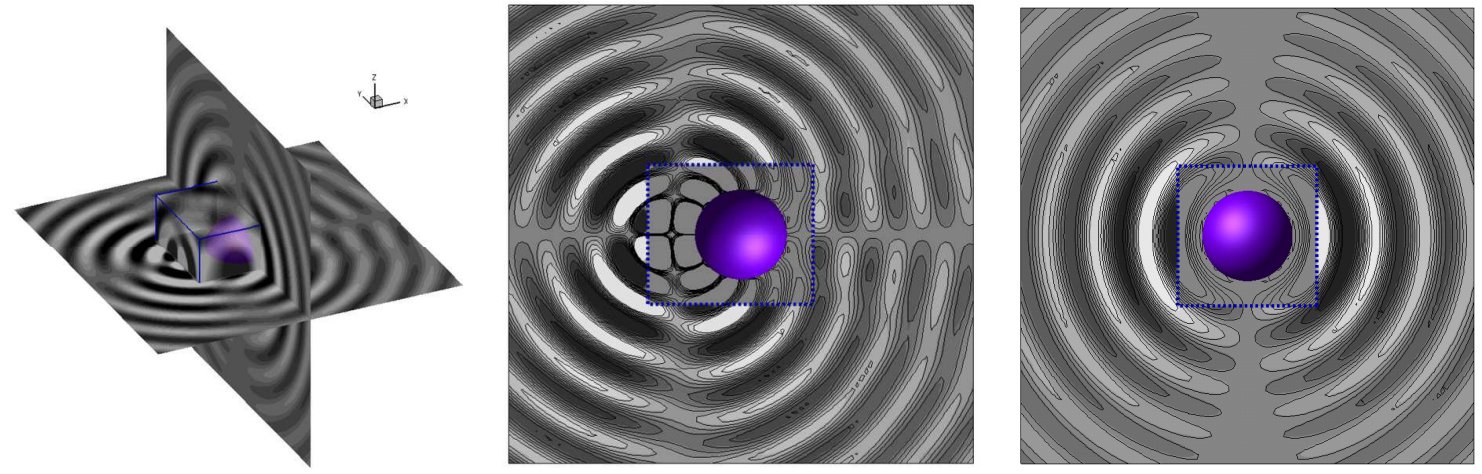

Figure 4. Validation of the Non Reflective Interface (NRI), through a test case excerpted from BANC Category 8.1.

Scattering of a harmonic $(k R=2 \pi R)$ quadrupole by a $3 D$ rigid sphere within a semi-infinite medium at rest, via an analytical-CAA hybrid calculation. Instantaneous perturbed pressure field obtained within either the xy (left and center) or the yz (left and right) median planes. Comparison of the NRI-forced CAA calculation results (in flood) against analytical ones (drawn in flood over the cubic envelope on left side, plotted in lines on center and right sides). Sphere is not CAAaccounted for and NRI interface appears in blue dashes. Analytical stage: BANC Cat. 8.1 (JAXA, Dr. Ikeda), CAA stage: sAbrinA solver (Onera).

In a second time, with the view of validating further the non reflective character of the NRI technique, the previous test case was slightly modified, the infinite space being replaced by an as-like semi-infinite one. This was achieved by prescribing a rigid plane over a lateral side of the computational box, as depicted in Fig. 5 (where the rigid plane appears in red colour).
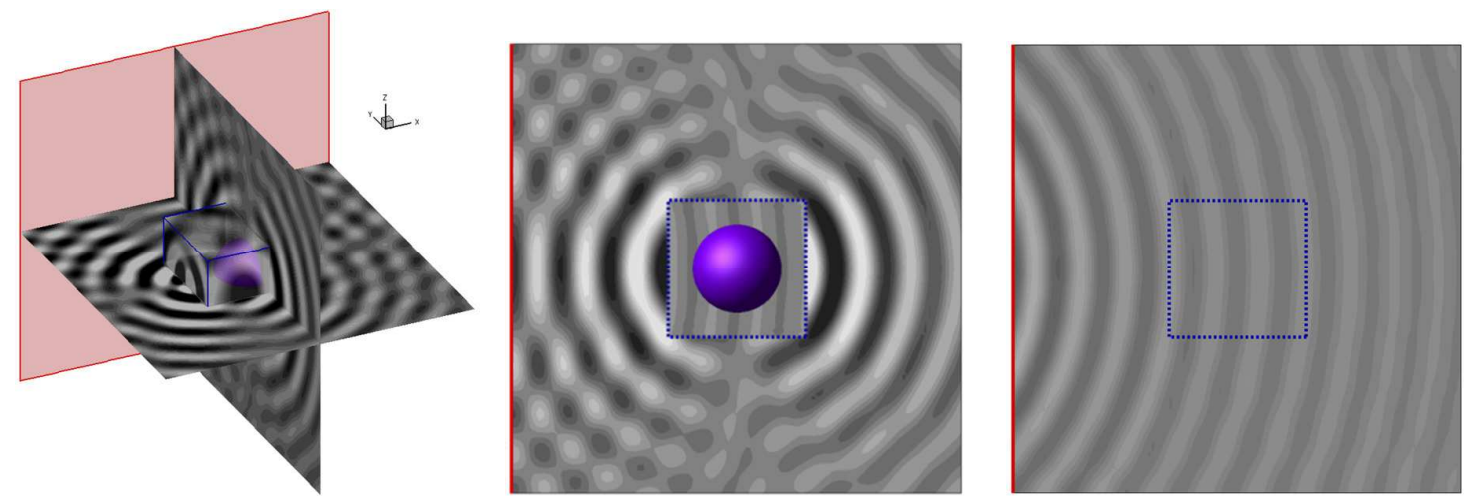

Figure 5. Validation of the Non Reflective Interface (NRI) ), through a test case derived from BANC Category 8.1. Scattering of a harmonic $(k R=2 \pi R)$ quadrupole by a $3 D$ rigid sphere within an as-like semi-infinite medium at rest, via an analytical-CAA hybrid calculation. Instantaneous perturbed pressure field obtained within xy (left and right) and yz (left and center) planes. Right side; scattered field induced by the rigid plane (in red). Sphere is not CAA-accounted for, NRI interface appears in blue dashes. Analytical stage: BANC Cat. 8.1 (JAXA, Dr. Ikeda), CAA stage: sAbrinA solver (Onera).

Figure 5 displays the instantaneous pressure field obtained after 15 periods of source emission, a time necessary for the acoustic field to reach a stationary state all over the domain. By comparing left sides of Figures 4 and 5, one can clearly observe the acoustic interaction patterns (such as standing waves, etc.), which translate the backscatter effects by the rigid wall (only, since the sphere was still not CAA-accounted for here). The latter effects are more visible in right side of Fig. 5, which depicts the sole scattered field. Please, note that the latter was obtained by subtracting from the total perturbed field computed here the incident one, which was derived from the previous 'infinite medium' calculation results (see Fig. 4). When looking at this scattered field, one can clearly distinguish how the acoustic waves were here first reflected by the rigid plane, before they were backscattered within the computational domain. On that stage, one can appreciate how such backscattered field is perfectly continuous over the entire calculation box, and more especially over the NRI interface (depicted in blue dashes). This confirms that the latter was not noticed by the backscattered waves, which validates the non reflective nature of the NRI technique. 


\section{Application and Further Validation of NRI through BANC-II Category 8.2}

As shown below, in a second time, the NRI-based CFD-CAA weak-coupling methodology was applied to a more realistic problem of aerodynamic noise; being directly inspired from the BANC-II Category 8.2 test case, this problem addressed the noise emission by two cylinders in tandem, with the latter being installed in the anechoic and so-called Quiet Flow Facility of NASA/LaRC (see Fig. 6, left side).

Indeed, to better understand some of the generic physical mechanisms associated with
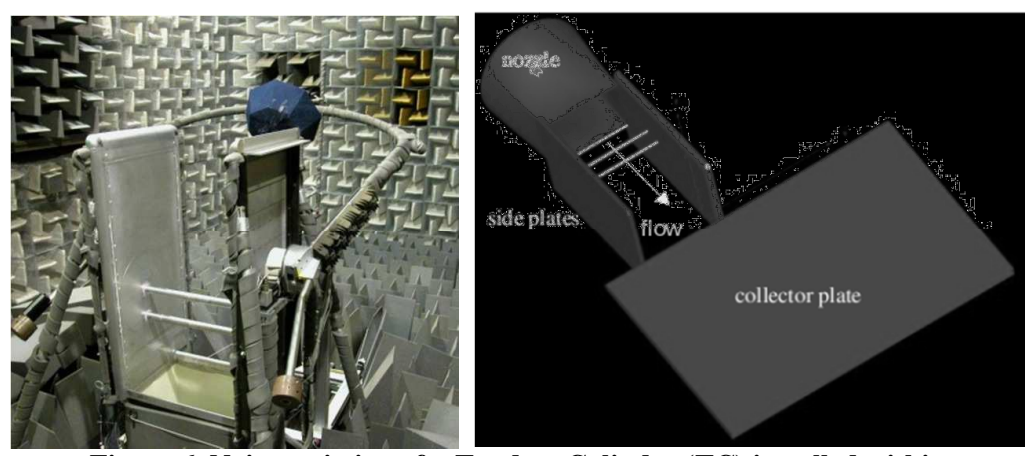

Figure 6. Noise emission of a Tandem Cylinder (TC) installed within NASA/LaRC's Quiet Flow Facility (QFF). Left side: TC model, with some of the QFF devices (nozzle, mounting side plates). Right side: sketch of the whole installed TC set-up, with all the QFF devices (nozzle, mounting side plates, collector plate). Reproduced from [15] with permission (courtesy of NASA).

the aeroacoustics of aircraft undercarriage systems, a combined experimental and computational campaign ${ }^{18-20}$ was carried out at NASA Langley Research Center (LaRC), focusing on both the aerodynamics and the acoustics of a Tandem Cylinder (TC) configuration. The model geometry is defined by two identical cylinders, spatially separated in the streamwise direction of an incoming flow. Such a simplified configuration constitutes an ideal test case for investigating the physics of aerodynamic noise associated with component interaction, as well as validating high-fidelity numerical prediction tools for similar sources of noise in actual aircraft systems (landing gears, etc). To this end, extensive experimental data had been collected ${ }^{18,19}$ and compared to the results of $3 \mathrm{D}$, unsteady compressible CFD computations ${ }^{20}$ (see top/left of Fig. 7), which had then been extrapolated to the farfield with the help of an Integral Method (IM) based on a $\mathrm{FWH}^{3}$ technique (see top/right of Fig. 7, in black dashes). Despite the favourable comparison between the measured and computed results, a legitimate concern existed about some of the obvious differences occurring between the installed TC configuration that had been tested and the simplified configuration that was computed. In other words, questions arose about the fact that these CFD-FWH hybrid calculations did not incorporate any of the possible installation effects that could have occurred in the experiments. Indeed, accounting for all or part of the facility environment (see Fig. 6) during the initial CFD stage would have been far too expensive, requiring a fine mesh to compute not only the cylinders, but also the side walls supporting and surrounding them. On the other hand, as was said above, the intrinsic limitations of Helmoltz-based integral methods ${ }^{1-3}$ limited the FWH stage from correctly accounting for the reflections / diffraction and refraction effects that may have been caused by the QFF itself and its confined jet flow.

Therefore, a dedicated study was conducted, in order to numerically assess the various acoustic installation effects that could have been effectively induced by the experimental set-up onto the acoustic data gathered during NASA/LaRC experiments. In particular, it was aimed at investigating not only the effects induced by the solid devices characterizing the experimental apparatus in the anechoic facility (see right side of Fig. 6), but also by its corresponding (confined and sheared) jet flow. These requirements could obviously be fulfilled by a CFDCAA hybrid calculation relying on the NRI technique, especially considering the latter would enable to properly handle all acoustic backscattering effects that were expected to occur, due to the QFF apparatus. Consequently, several CFD-CAA hybrid calculations of the QFF-installed TC configuration were performed ${ }^{13}$, all being performed using the NRI-based CFD-CAA weak-coupling procedure, which was fed with an identical CFD dataset. The latter was derived from the isolated TC unsteady CFD computations evocated above, for which near-field data had been obtained using NASA/LaRC's CFL3D solver (that had been run with a hybrid RANS/LES turbulence model - see Ref. 20).

First, a preliminary CFD-CAA calculation was conducted, for which the TC configuration was considered as isolated, i.e. free of any installation effects. For such an isolated case, the steady mean flow imposed in the CAA calculation was derived in accordance with the CFD one, corresponding thus to a 'free flow' configuration. Such a preliminary calculation first allowed validating further the NRI-based CFD-CAA surface coupling approach, through a direct comparison of results with the ones initially obtained via the CFD-FWH hybrid approach ${ }^{20}$ (see for instance the associated far-field noise spectra in right top of Fig. 7, drawn in black lines and dashes respectively). In addition to that, such preliminary calculation provided a reference solution against which comparing the results obtained from the subsequent installed-TC calculations, in order to assess the various effects characterizing the QFF facility environment. These installed-TC calculations were performed with a different degree of realism included each time, so as to estimate separately the reflection / diffraction effects of each component (side plates, collector plate, nozzle) of QFF environment, as well as the convection / refraction 
effects by its confined and sheared jet flow.
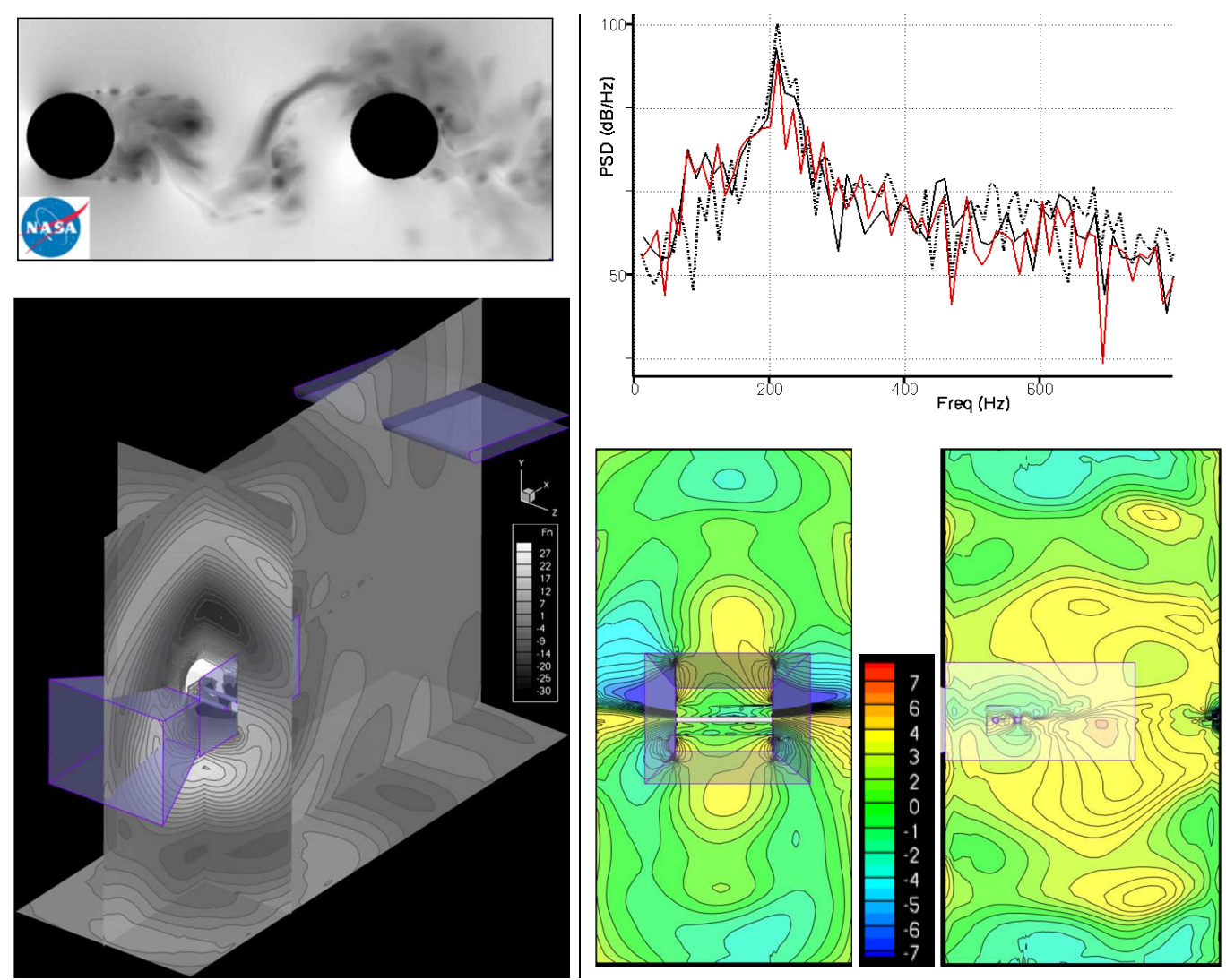

Figure 7. Validation / application of the NRI-based CFD-CAA weak-coupling procedure and associated hybrid approach, through a test case derived from BANC-II Category 8.2. Noise emission of a Tandem Cylinder (TC) installed within NASA/LaRC's QFF anechoic facility, via CFD-CAA hybrid calculations. Left side: instantaneous perturbed fields obtained via both a CFD calculation of the isolated TC (top, courtesy of NASA) and a CFD-CAA computation of the QFFinstalled TC (bottom). Right side, bottom: deltas (in dB) between the Sound Pressure Level fields associated with the isolated and the QFF-installed configurations, as recorded within two lateral planes (xy and yz). Right side, top: Power Spectral Density of the acoustic pressure radiated in the far-field (QFF-installed TC in red, isolated TC in black. In black dashes, isolated TC results obtained via a CFD-FWH method). CFD stage: CFL3D solver (NASA, Dr. Lockard), CAA stage: sAbrinA code (Onera).

As an illustration, left bottom of Fig. 7 displays an instantaneous snapshot of the perturbed pressure field delivered by the CFD-CAA calculation associated with the fully installed TC configuration, which included both the QFF facility main devices and the corresponding confined / sheared jet flow. Such results are compared against the ones obtained for the isolated configuration, with respect to either the far-field noise radiation (see the red and black spectra, in right top of Fig. 7) or the near-field acoustic propagation (see the delta effects between the two respective Sound Pressure Level maps, in right bottom of Fig. 7). More details about this study, which results are too numerous to be discussed here, can be found in Ref. 13.

\section{Optimization of the CAA-based Hybrid Approach, and Validation/Application through Test Cases from BANC Category 8.1}

\section{A. Optimization of the CAA-based Hybrid Approach}

At that stage however, it was needed to optimize further the overall hybrid approach, so that it can handle still more complicated problems (e.g. those involving very complex aeroacoustic signals and/or geometries). In particular, one major objective was to assess and/or improve the way such hybrid procedure could (i) cope with all stringent constraints that are dictated by real-life applications (ii) without being jeopardized by some of their unavoidable side-effects (such as the signal degradation to which unsteady CFD data are subjected, when manipulated for being acoustically exploited ${ }^{22}$ ). With the view of answering those key questions, several R\&D actions were conducted by the present authors, being primarily achieved by the second one in the framework of his $\mathrm{PhD}$ thesis ${ }^{21}$. The objective here was to (i) characterize and (ii) possibly minimize the various impacts that its 
data manipulation might have onto acoustic signals to be transferred from one to another stage of an aeroacoustic hybrid method. These actions made it possible to deliver specific fundamental insights and to propose several innovative solutions (see below), for hybrid methods can be optimized. Regarding more specifically the present CAA-based hybrid approach, these outcomes were numerically illustrated and validated on the basis of various academic test cases of increasing complexity, among which the problem excerpted from BANC Category 8.1 (acoustic scattering by a 3D sphere in a medium at rest, see section V.B below). All these outcomes were then applied to a real-life problem directly inspired from BANC-III Category 8.2 (noise emission by a nose landing gear, see section VI below), for which they allowed to derive an optimal CAA computational set-up, as well as to perform properly the $\mathrm{CFD} \rightarrow \mathrm{CAA}$ data transfer operations.

\section{A.1 Minimization of the Signal Degradation Induced by the CFD Data Manipulation}

First, theoretical studies were conducted in order to address the signal degradation that unsteady CFD data may be subjected to, when sampled and/or interpolated in space and/or time ${ }^{22}$. Indeed, hybrid calculations generally require that the aeroacoustic signal to be transferred from one to the other stage is submitted to a certain number of manipulations; first, since hybrid calculations are usually not conducted simultaneously, the unsteady data that are computed at a given stage must be stored before they are transferred to the next hybrid step. Such operation is often conducted with a space and/or time sampling applied, so that the storage time and memory requirements are minimized. Second, because of the mismatches that may occur between the various discrete formulations ruling each one of the stages to be weakly-coupled, when transferred from one to another stage, these data must then be interpolated in time and/or space. The point is that both the sampling and the interpolation operations are subjected to side effects that may lead to a complete degradation of the aeroacoustic signal to be transferred, which suffices to question the accuracy and robustness of the resulting overall hybrid procedure. For instance, theoretical investigations ${ }^{22}$ conducted in the present framework highlighted how far the acoustic information delivered by a given CFD calculation can be dramatically and irremediably degraded, depending on the way CFD data are effectively stored and/or processed for being acoustically exploited. Among other things, there, it was shown how their sole sampling (in space and/or time) may degrade the CFD datasets as much - and sometimes even more - than their interpolation. More important, these theoretical investigations helped in better understanding and modelling the two intrinsic mechanisms that are responsible for the signal degradation, which are the aliasing and the spuriousing phenomena ${ }^{22}$. This led to derive a dedicated formalism ${ }^{22}$ that allows predicting a priori i) when and how a given unsteady signal may be degraded when sampled and/or interpolated, as well as ii) how far such degradation may then impact the acoustic propagation stage (whether the latter is of CAA or IM type). With the view of minimizing such impact, innovative solutions were then developed; among other things, one can mention the so-called Interpolation By Part (IBP) technique ${ }^{23,14}$, which allows interpolating accurately a given signal, while minimizing its possible degradation. On the same way, one can mention the specific Signal Preservation Criteria, which can serve as general guidelines for adjusting the various elements (CFD storage, CAA stage, etc.) of a given CFD-CAA hybrid scenario, so that the aeroacoustic signal to be $\mathrm{CFD} \rightarrow$ CAA transferred is preserved at best. On that stage, one can underline that the fundamental insights and methodological outcomes that were gathered within this framework are not restricted to the sole case of an aeroacoustic hybrid method relying on a CAA noise propagation stage. Indeed, most of the theoretical results and/or innovative techniques developed there could easily be extended to other aeroacoustic hybrid approaches (e.g. those relying on an IM noise radiation stage), since they are also subjected to the same kind of side-effects (signal degradation, etc.). On the same way, beyond their application to hybrid methods, many of the previous outcomes could also be advantageously applied to research areas involving interpolation techniques (e.g. multisize-mesh multi-time-step problems, immersed boundary methods, overlapping grid techniques, etc.).

\section{A.2 Relaxation of the Constraints Weighting on both the CFD Data Transfer and the CAA Stage}

In a second time, and for tentatively coping with all restrictive constraints imposed by the necessary preservation of the aeroacoustic signal to be transferred from one stage to the other, several improvements were brought to the present hybrid approach. First, with the view of minimizing the sampling of CFD data to be stored (by decreasing their overall volume), the NRI technique was optimized, so that its minimal storage requirements could be relaxed. This was achieved by adapting the NRI process so that it can be handled by space operators of reduced stencil sizes, compared to those used for the propagation stage (see Ref \#7). Once validated on the basis of the test case excerpted from BANC Category 8.1, this optimized version of the NRI was applied to the one coming from BANC-III Category 8.2 (nose landing gear problem, see section VI below), allowing to reduce by one half the volume of unsteady CFD data to be CAA-exploited.

Second, with the view of reducing at maximum the CAA grid density (and, thus, volume) to be handled for the propagation stage, a new class of finite difference (FD) propagation schemes was developed ${ }^{24}$. As detailed in Ref. 21, these so-called Intrinsically Optimized FD (IOFD) schemes are of optimal accuracy, thanks to an optimization process that is based on a minimization of the scheme's leading-order truncation error (rather than on an optimization of the scheme' spectral properties, such as usually done ${ }^{25-27}$ ). Thanks to their as-like optimal 
accuracy, these IOFD schemes allow dealing with CAA grids of much coarser nature; for instance, with no more than 4 (resp. 6) Points Per Wavelength (PPW), an IOFD scheme of 15 (resp. 11) points guarantees that the error made on the group velocity (on which depends the acoustic energy transport and, thus, the overall accuracy of the CAA stage) is less than $0.1 \%$. Compared to a classical 7 -point $/ 6^{\text {th }}$ order standard finite difference scheme (which corresponding minimal PPW is 12), this represents a gain of 3 (resp. 3/2) per direction, i.e. a factor 27 (resp. 3.375) in 3D. Such a benefit can allow either a reduction of the computational effort to be paid, or an increase of the maximal frequency to be reached during the CAA stage (with memory requirements that go decreasing by the same factor - while CPU times can be reduced even more, e.g., down to a factor 54, depending on the CFL constraints). As a corollary, regarding the present CAA-based hybrid approach and weakly-coupled CFD-CAA calculation matters, such a reduction of the CPU/MEM needs by the CAA stage can be directly turned into a beneficial increase of the CFD storage density - which, at the present date, constitutes the best way for minimizing the signal degradation to be possibly induced by the manipulation of CFD data. As a practical example, one can here notice that the test case derived from BANC-III Category 8.2 (nose landing gear, see section VI below) was numerically handled with the help of 15-point IOFD schemes, allowing then to deal with a 27 times lighter CAA grid volume and, thus, calculation CPU time, compared to the one(s) that would have been required if a regular 7-point standard FD scheme had been used instead. This made it possible to not only i) reduce the overall CPU/MEM price paid for the CAA stage, but also to ii) increase the mesh density of the $\mathrm{CFD} \rightarrow$ CAA weak-coupling area and, thus, the accuracy of the CFD $\rightarrow$ CAA data transfer operations (space sampling and interpolation tasks).

\section{B. Validation of the Optimized CAA-based Hybrid Approach through BANC Category 8.1}

All the previous outcomes were validated on the basis of various academic test cases of increasing complexity, among which the BANC Category 8.1 problem introduced above (acoustic scattering by a 3D sphere in a medium at rest). To this end, as shown below, the calculation case detailed at section IV.B was conducted again, after its computational set-up was modified in regard to several aspects (acoustic source contents, numerical schemes, etc.) so that each one of the various outcomes can be properly validated.

\section{B.1 Validation of the Interpolation By Parts (IBP)}

First task aimed at highlighting i) how far acoustic signals may be degraded when manipulated (i.e. sampled and/or interpolated) in time, and ii) how the innovative Interpolation By Part (IBP) process can help in partly remedying such issue. For doing so, the calculation case of section IV.B was repeated as is, to the exception that the acoustic source signal originally defined $(k R=2 \pi R)$ was here combined with an extra noise of same amplitude, but of much higher dimensionless frequency $(k R=9 \pi R)$. Once cumulated, both components resulted in a single signal $(k=2 \pi+9 \pi)$, which was discretized in time, as if the signal had been stored at every one tenth of the primary emission source period $\left(\Delta \mathrm{t}_{\text {Storage }}=T_{2 \pi} / 10\right)$. This signal was then time-interpolated for fitting a CAA calculation time step that was two third times smaller than the storage one $\left(\Delta \mathrm{t}_{\mathrm{CAA}}=2 / 3 \Delta \mathrm{t}_{\text {Storage }}\right)^{*}$. Thanks to the theoretical analysis tools developed in the present framework, one could predict that the manipulation (i.e. time sampling + interpolation) of such signal would lead to the generation of various spurious modes, of respective wavenumbers corresponding to multiples of $\pi\left(k_{\text {spurious }}=m \pi, m \in Z\right)$. The interpolation stage was carried out either with the help of a classical approach (via a centered $4^{\text {th }}$-order Lagrange interpolator, L4), or through the Interpolation By Part technique (IBP4, based on L4 interpolator - see Ref. 23 for more details). Each one of the two interpolated signal was then NRI-forced and CAA-propagated, its resulting time signature being recorded at four different locations of the CAA domain (M1 to M4, see the sketch on left side of Fig. 8) and, then, spectrally decomposed by the means of a Fourier analysis; right side of Fig. 8 plots the spectra delivered by each signal, comparing it to the analytical solution corresponding to the sole primary signal (i.e. $k=2 \pi$ ). As one can see in these plots, whatever the nature of the interpolation to be applied was, the cumulated signal $(k=2 \pi+$ $9 \pi)$ saw its lower frequency mode of interest $(k=2 \pi)$ being well preserved by the data manipulation, CAAforcing and propagation. On another hand, and although it was partly altered by it, the higher frequency mode $(k$ $=9 \pi$ ) was not totally dissipated by the CAA stage ${ }^{\dagger}$. More important, for both signals, the spectra exhibited additional tones, which corresponded exactly to the spurious modes that were expected to occur, because of the signal manipulation. Such spurious contents, however, were more importantly filtered out by the interpolation stage when the latter relied on an IBP procedure than when it was based on its standard Lagrange interpolator counterpart (see the respective levels of blue and red components, in right side of Fig. 8).

\footnotetext{
* One can notice here that such a configuration (which differs from the one addressed in section IV.B, for which one had implicitly $\Delta t_{C A A}=\Delta t_{\text {Storage }}=T_{2 \pi} / 20$ ), is quite typical of what can be encountered in real-life applications, for which the CAA time step is generally smaller than the CFD-storage one.

${ }^{\dagger}$ This indirectly proves that, once created, high frequency - and possibly insufficiently resolved - signals may not be so easy to filter out from the CAA stage.
} 

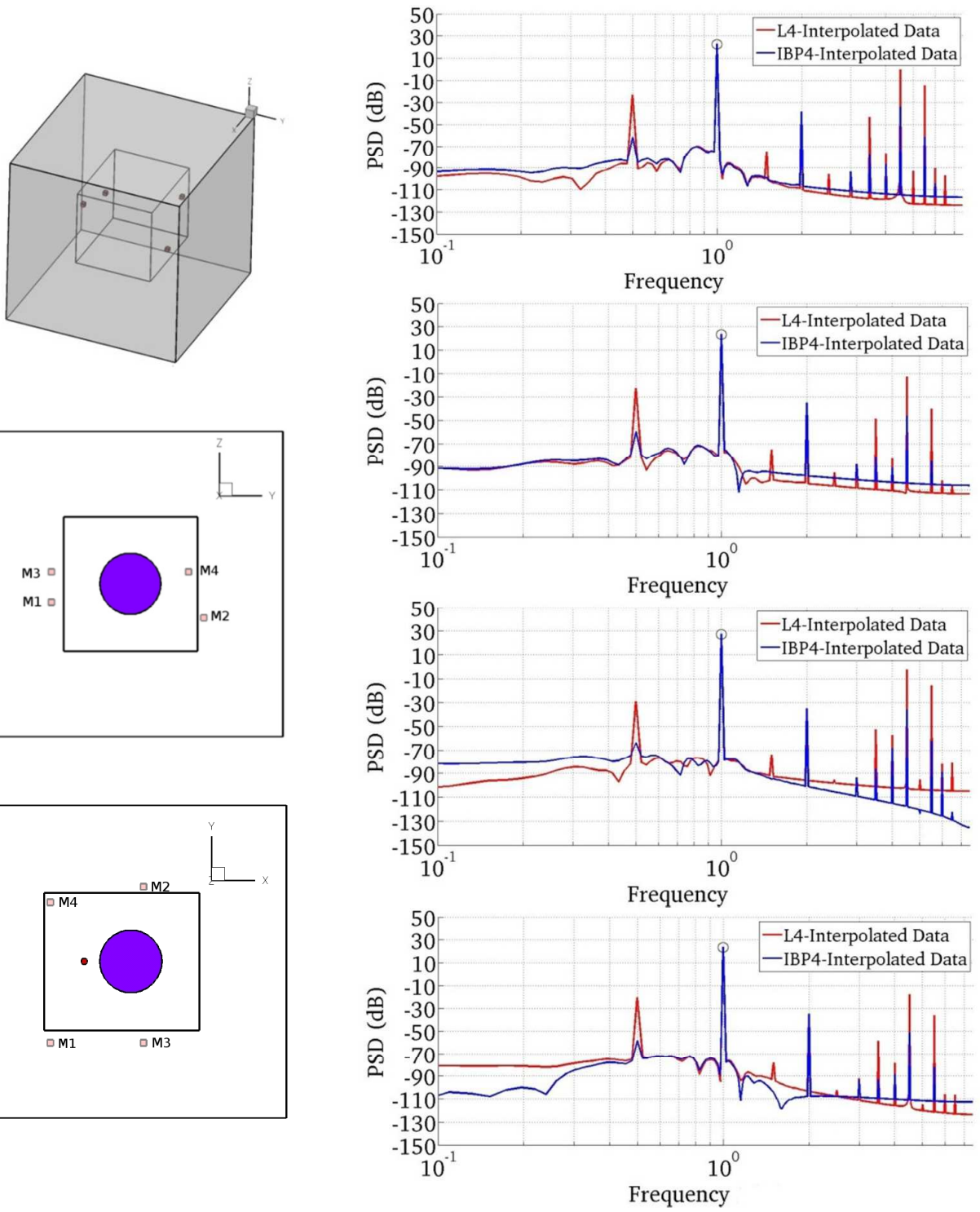

Figure 8. Validation of the Interpolation By Parts (IBP), through a test case derived from BANC Category 8.1.

Scattering of a two-tones $(k R=2 \pi R+9 \pi R)$ quadrupolar emission by a $3 D$ rigid sphere within an infinite medium at rest, via analytical-CAA hybrid calculations. Left side: Sketch depicting the CAA calculation box, the source region (central cubic zone), the quadrupole location (red spot) and the four microphones locations (M1 to M4). Right side: Power Spectral Density of both the manipulated (time sampled + interpolated) and the NRI-forced/CAA-propagated signal, as recorded for microphones M1 to M4 (from top to bottom). The black circle indicates the (analytical)spectra associated with the low frequency tone $(k R=2 \pi R)$. Analytical stage: BANC Cat. 8.1 (JAXA, Dr. Ikeda), CAA stage: sAbrinA solver (Onera).

This validation tasks demonstrated how far the Interpolation By Part technique can help in partly remedying to the signal degradation inherited from the manipulation of unsteady data to be transferred from one stage to the other of an aeroacoustic hybrid method.

\section{B.2 Validation of the Intrinsically Optimized Finite Difference (IOFD) schemes}

Second validation task aimed at demonstrating how IOFD schemes effectively offer to enhance the accuracy of the CAA stage (and, thus, the preservation by the latter of acoustic signals it is forced with), to the direct benefit of the overall hybrid calculation. With that view, the calculation case of section IV.B was repeated as is, to the exception that the acoustic source signal originally defined $(k R=2 \pi R)$ was then replaced with a signal of same amplitude, but of higher dimensionless frequency $(k R=6 \pi R)$. With respect to the present computational set-up (which CAA grid was kept the same), such signal was thus discretized with no more than 3.3 PPW (Points Per Wavelength); thanks to the theoretical analysis tools developed in this particular framework, one could predict that, even without being manipulated (i.e. sampled and/or interpolated in time and/or space), such signal would generate spurious numerical noises from the moment it would be CAA-handled with derivative operators of 
insufficient accuracy. Four calculations were thus conducted, each one being performed with a particular derivative operator, to be based on standard finite differences (SFD) schemes of either 7-point (resp. $6^{\text {th }}$ order) or 11-point stencil (resp. $10^{\text {th }}$ order), as well as on intrinsically optimized finite difference (IOFD) schemes of either 11-point (resp. $6^{\text {th }}$ order) or 15 -point stencil (resp. $8^{\text {th }}$ order). For all calculations, the filtering stage was handled with a very high fidelity filter (SFD of 21-point stencil / $20^{\text {th }}$ order), that is, ensuring a negligible bias on the propagation results. Finally, regarding the CAA-forcing, the NRI procedure was achieved with suitable interfaces (i.e. of possibly thicker envelopes, compared to the baseline computational set-up of previous cases).
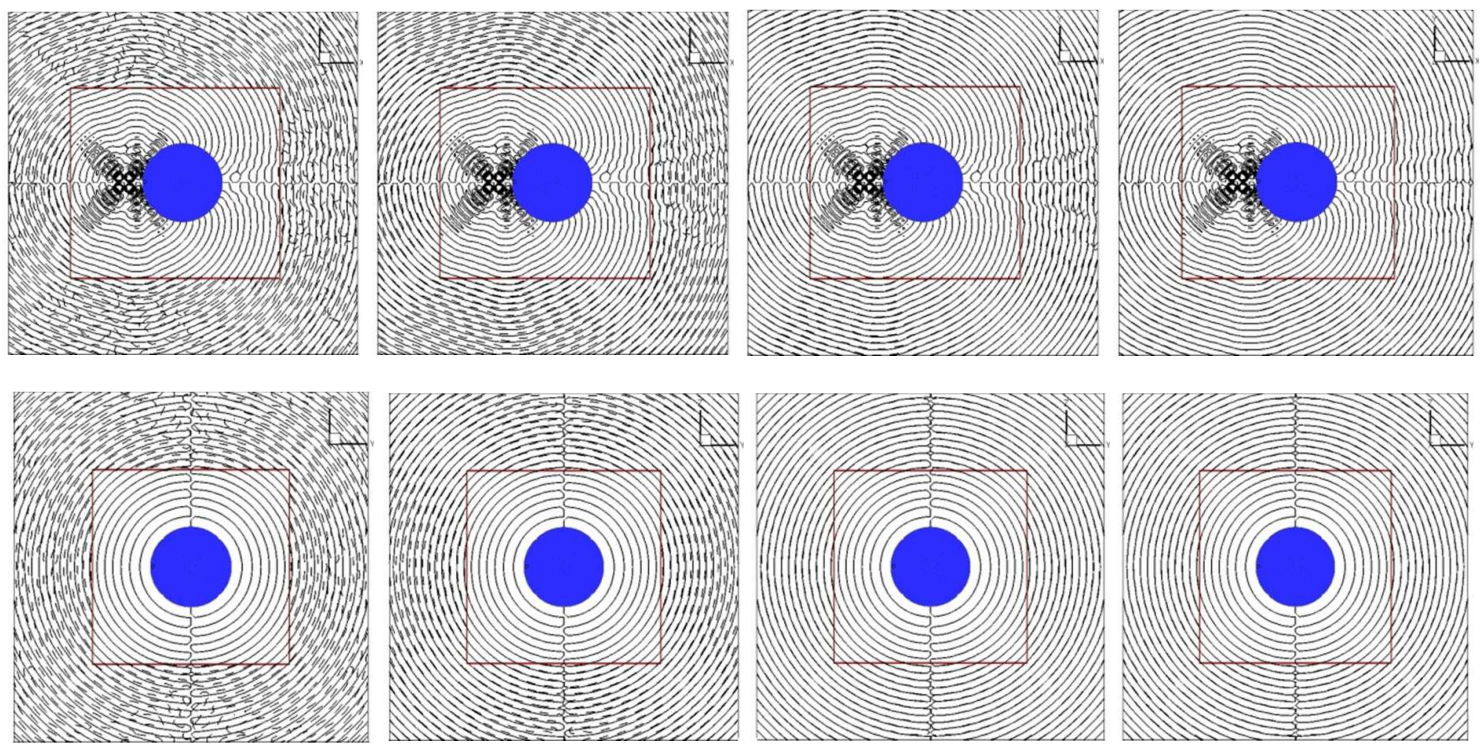

Figure 9. Validation of the Intrinsically Optimized Finite Differences (IOFD) schemes, through a test case derived

from BANC Category 8.1. Scattering of a very high frequency $(k R=6 \pi)$ acoustic quadrupole by a $3 D$ sphere within a quiescent medium at rest, via analytical-CAA hybrid calculations. From left to right: standard FD (SFD) schemes of 7- and 11-point stencils, intrinsically optimized (IOFD) schemes of 11- and 15-point stencils. Comparison of numerical outputs (in dashes, out of the source zone / red box) against analytical data (in line, over the entire domain), as obtained within either the xy (top) or the yz (bottom) plane. Analytical step: BANC Cat. 8.1 (JAXA, Dr. Ikeda), CAA stage: sAbrinA solver (Onera).

Figure 9 depicts the four calculation results, displaying for each one the instantaneous perturbed pressure field obtained (out of the source zone) at the end of the computation, and comparing it to the analytical results (depicted all over the computational box). As one can see, both standard schemes (SFD) failed in propagating properly such a high frequency signal, generating spurious modes that corrupted the acoustic field all over the domain. On the contrary, however, both intrinsically optimized schemes (IOFD) behaved much better; indeed, as one can see, the 11-point stencil $/ 6^{\text {th }}$ order IOFD scheme delivered an acoustic field that was almost free of spurious modes, whereas its counterpart of 15-point stencil $/ 8^{\text {th }}$ order counterpart provided a result matching exactly the analytical one. This demonstrates how far IOFD schemes effectively offer an advantageous alternative to standard ones, allowing enhancing the accuracy of the CAA stage, and thus, to preserve at best the aeroacoustic signal to be transferred to / propagated by the latter. Here, one can notice that the present exercise made it possible to check that, compared to the 7-point $/ 6^{\text {th }}$ order SFD (which is the most popular FD scheme used among the CAA community), an IOFD scheme of 15-point stencil $/ 8^{\text {th }}$ order (resp. 11-point $/ 6^{\text {th }}$ order) requires no more than $50 \%$ (resp. $25 \%$ ) extra CPU time per operation, while it allows the CAA grid to be coarsen by a factor of 3 (resp. 3/2) per direction ", offering thus to lower the mesh density by a factor 27 (resp. 3.375 ) in 3D. Again, because of CFL aspects, these numbers can be multiplied by up to 2, when it comes to consider the CPU time reduction offered by such a grid density decrease.

\section{Application of the Optimized CAA-based Hybrid Approach through Test Case from BANC-III Category 8.2}

Finally, all the previous outcomes and associated developments (NRI, IBP, IOFD, etc.) were further validated and illustrated through an application to a realistic aircraft noise problem, i.e. the noise emission by a nose landing gear. Being addressed in BANC-III Category 8.2, such test case was derived from the so-called LAGOON project (LAnding Gear nOise database for CAA validatiON), which was supported by Airbus and

\footnotetext{
${ }^{*}$ Considering that the 7-point stencil standard FD, the 11-point stencil IOFD, and the 15-point stencil IOFD schemes are characterized by an accuracy limit of $\pi / 6, \pi / 4$ and $\pi / 2$, respectively.
} 
conducted by several partners (Onera, DLR, Southampton University, etc.), so as to validate accurately the computational methods dedicated to landing gear noise prediction. Within the latter framework, an experimental campaign was first carried out at Onera, focusing on both the aerodynamics and the acoustics of a simplified landing gear geometry ${ }^{*}$ which was considered in an isolated configuration and allotted
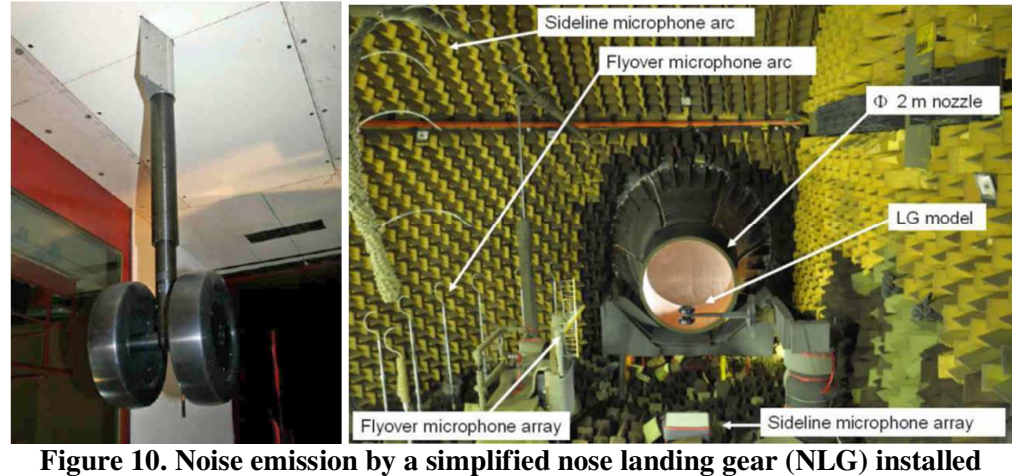

Figure 10. Noise emission by a simplified nose landing gear (NLG) installed within Onera's facilities. $N L G$ model (seen from behind), as installed in Onera's F2 aerodynamic facility (left) and CEPRA19 open jet anechoic wind tunnel (right). Reproduced from [31] with permission (courtesy of Airbus).

either a take-off or an approach

flight condition. These aero+acoustic dual experiments ${ }^{28,29}$ were achieved in Onera's aerodynamic $(F 2)$ and anechoic (CEPRA 19) facilities, respectively (see Fig. 10). Lately, part of the experimental database was made available to the aeroacoustics community, being constituted as the fifth category of the BANC initiative. In a second time, the computational counterpart of such aero+acoustic experimental campaign was conducted at Onera; aerodynamics computations relied on 3D unsteady compressible CFD calculations (based on the socalled $z D E S$ approach), which were all conducted thanks to Onera's solver named $e l s A^{30}$. As one can see in top right side of Fig. 11, those calculations were favourably compared with the aerodynamic measurements through
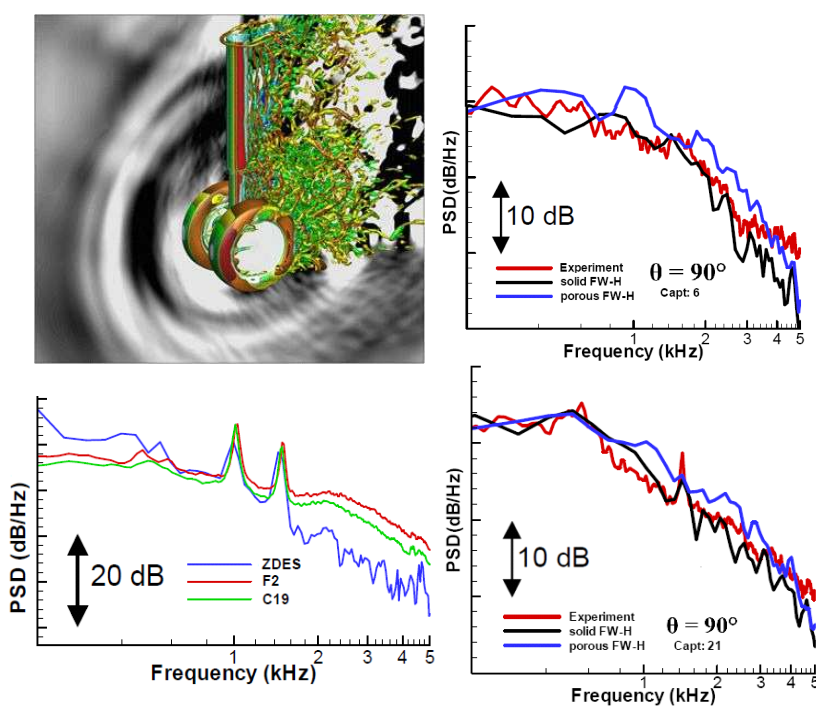

Figure 11. Aeroacoustics of the NLG in isolated configuration (i.e. in free-field), as predicted by a CFD-FWH hybrid calculation. Left side: near-field aerodynamic results (top: $Q$-criterion iso-surfaces colored by the stream wise velocity component and instantaneous pressure fluctuation field), with validation (bottom) via direct comparison of the Power Spectral Density (PSD) computed by CFD (in blue) and recorded in the experiments (in red and green), for a probe of the right wheel. Right side; validation of the far-field acoustic results, via direct comparison of the PSD predicted by CFDFWH (black and blue) and measured in the experiments (red), for two microphones located at $90^{\circ}$ from the model in the flyover (top) and side line (bottom) directions. CFD stage: elsA solver (Onera, Dr. Ben Khelil). FWH stage: KIM code (Onera, Mr. Sanders). Reproduced from [31] with permission direct comparison of near-field results ${ }^{\dagger}$. In particular, both experimental and numerical outputs exhibited tonal noises (of approx. $1 \mathrm{kHz}$ and $1.5 \mathrm{kHz}$ ), which emission was inferred to be associated with resonances coming from the wheels' inner cavities. These unsteady CFD results were then acoustically extrapolated to the far-field ${ }^{31}$ via an Integration Method (IM) based on a $\mathrm{FWH}^{3}$ approach and solver (Onera's code $\mathrm{KIM}^{32}$ ). As one can see in right / bottom of Fig. 11, these CFD-FWH hybrid calculation results were also favourably compared to the experimental measurements recorded in the far-field (this, to the exception of mismatches coming from side-effects induced by the FWH stage, depending on the integration technique used ${ }^{\frac{1}{*}}$ ).

Despite of the good match between numerical and experimental outputs, here too, it was decided to go further in such a hybrid prediction of NLG aeroacoustics, by improving the fidelity of its acoustic propagation stage; indeed, here, objectives were i) to account for the acoustic emission that had been effectively predicted by the CFD stage (rather than to model it via equivalent sources, as implicitly done in the FWH approach), as well as ii) to consider the realistic jet flow characterizing the experiment (rather than to model it via a simplistic

\footnotetext{
${ }^{*}$ Model geometry was that of a nose gear of an Airbus A320 aircraft, with a scale factor of 1:2.5, and with only the main elements (leg, wheels, etc.) kept.

${ }^{\dagger}$ To the exception of a mismatch over the low frequency range, which was assumed to come from a high pass filtering of experimental data.

With, for the so-called permeable (or porous) surface integration technique, numerical issues coming from the integration of non acoustical (hydrodynamic) occurrences convected by the landing gear's wake.
} 
uniform mean flow, as also done in the FWH approach). As for the previous TC case, these two requirements could be fulfilled by a CFD-CAA hybrid calculation relying on the NRI functionality. Therefore, two CFD-CAA hybrid calculations of the present NLG configuration were conducted ${ }^{16}$, both being achieved thanks to the NRIbased CFD-CAA weak-coupling procedure, which was fed with an identical CFD dataset (coming from that particular LAGooN unsteady CFD computation associated with a flow stream of Mach and Reynolds number $M$ $=0.18$ and $R e_{D}=1.210^{6}$, respectively). First CFD-CAA hybrid calculation focused on the isolated NLG, that is, its computational set-up was similar to the CFD-FWH computation one (incorporating in particular a steady mean flow corresponding to a homogeneous free field). Such a calculation offered to validate the present CFDCAA coupling exercise, through direct comparison against both the numerical (CFD) and the experimental results, which had been acquired and/or processed under the same conditions of a homogeneous medium ${ }^{*}$. In a second time, and following what had been done for the previous TC case, the CFD-CAA calculation considered the NLG as installed in the anechoic facility, with a (heterogeneous) background flow matching the confined and sheared mean jet occurring in the wind tunnel (see bottom left of Fig. 12). Such an alternative calculation made it possible to enhance further the fidelity of the prediction by CAA-accounting for the facility jet flow, as well as to assess the effects such jet flow may have had on the experimental measurements. The reader is referred to Ref. 16 for more details about this work, which only a few excerpts are provided below.
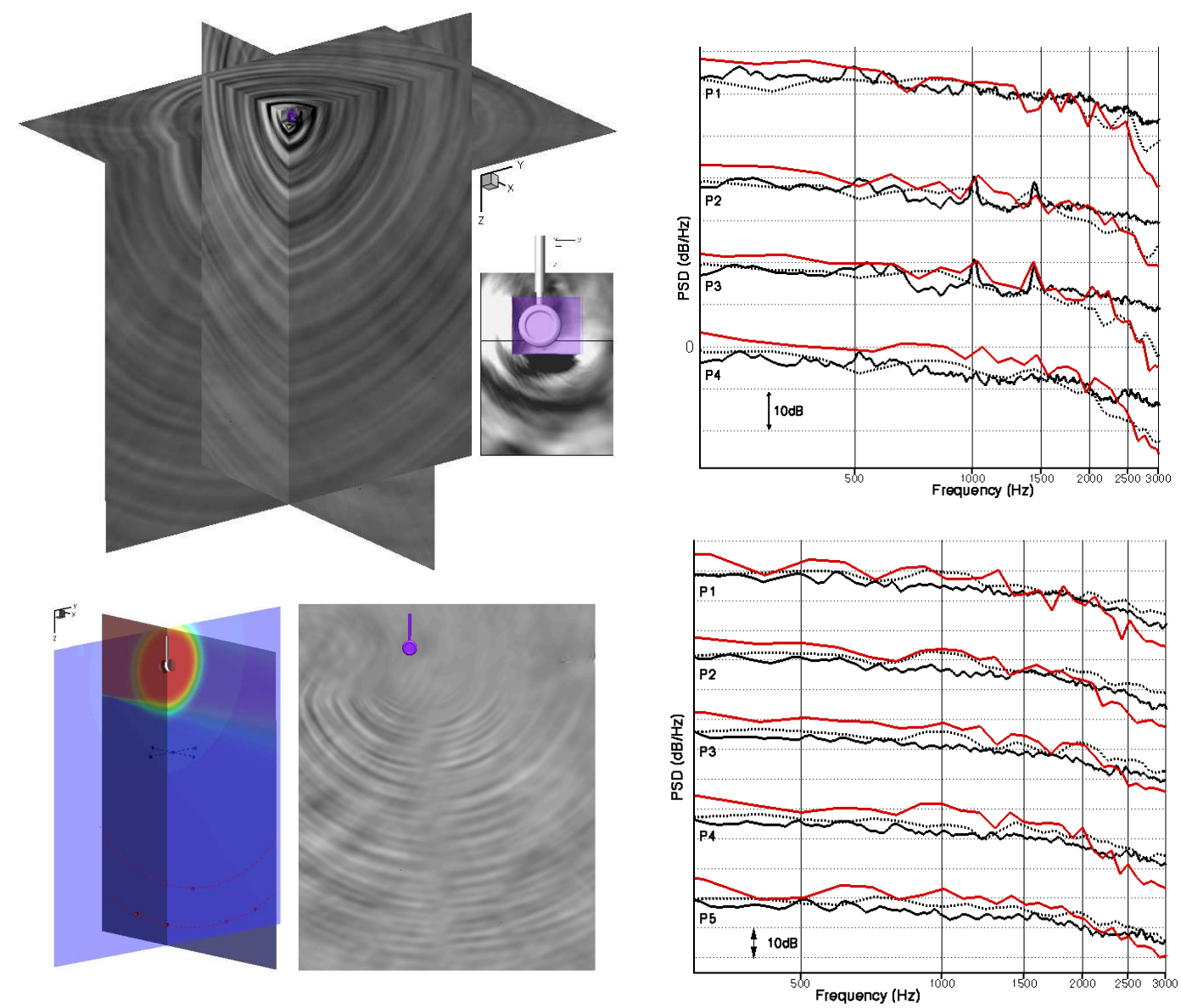

Figure 12. Validation / application of the optimized high fidelity acoustic hybrid approach, through a benchmark exercise derived from BANC-III Category 8.2. Noise emission of a Nose Landing Gear (NLG) installed within Onera's CEPRA19 anechoic facility, via CFD-CAA hybrid computations. Left: instantaneous perturbed field delivered by the 'installed NLG' calculation (top), facility confined/sheared steady jet flow (bottom left, axial velocity), and effects induced by the latter on the former (bottom right, deltas between the installed and the isolated configurations recorded within the $y$ plane). Right side; validation of the 'isolated $N L G$ ' calculation by cross-comparison of the spectra recorded within either the mid- (top) or the far- (bottom) fields for both the CFD-CAA (in red lines), the experiments (in black lines) and the CFD-FWH

(in black dashes). Mid-and far-field microphones are drawn (in blue and red, respectively) on the mean flow plot (left/bottom). CFD stage: elsA solver (Onera, Dr. Ben Khelil), CAA stage: sAbrinA code.

Figure 12 depicts the computational results of these two CFD-CAA hybrid calculations with, first, a

*Here, the experimental dataset had been corrected from the refraction effects by the open jet shear layers. 
snapshot of the instantaneous perturbed pressure field obtained at the end of the facility-installed NLG calculation (see top/left side of Fig. 12). As one can see, after it had been NRI-forced within the CAA domain through the weak-coupling interface (small cubic box, drawn here in purple), the CFD source signal was CAApropagated up to the far-field. One can notice that the resulting acoustic emission was somehow irregular, translating the intermittent character exhibited by the CFD source signal itself. In order to validate the present NRI-based CFD-CAA coupled calculations and underlying methodology, the results obtained for the isolated NLG configuration (i.e. associated with a free-field jet flow) were compared against the ones coming from either the preliminary CFD or the experiment; first, the CFD-CAA time signature and spectra were successfully compared against the ones that had been gathered during the CFD stage, such comparison exercise being conducted for six identical probes located in the immediate vicinity of the coupling interface. Such a near-field validation exercise was then extended to the mid (resp. far-field) region(s), where the spectra of the CFD-CAA signals were compared against the ones that had been experimentally recorded, this being achieved for 4 (resp. 5) probes located in the flyover direction, approx. 13R (resp. 40R) away from the mock-up (with $R$ standing here for the NLG wheel radius). Such mid- (resp. far-) field validation exercise turned out to be rather satisfactory, as one can see in top (resp. bottom) right side of Fig. 12, which compares the spectra delivered by both the experimental measurement (in black lines) and the CFD-CAA coupled calculation (in red lines) ${ }^{*}$. Note that, for indicative purpose, the spectra coming from the CFD-FWH (solid surface) hybrid calculations ${ }^{31}$ that had been initially achieved for the same isolated NLG configuration were also plotted here (in black dashes); as one can see, contrarily to the CFD-FWH ones, the CFD-CAA signals delivered in the mid-field reproduce fairly well the two tonal emissions that emerged from the experimental data measured in the gear's lateral directions (see for instance what happens at $1 \mathrm{kHz}$ and $1.5 \mathrm{kHz}$ for probes $\mathrm{P} 2$ and $\mathrm{P} 3$, in top/right of Fig. 12). Finally, the effects by the facility-installed jet flow onto the NLG acoustic emission appear in left bottom of Fig. 12, which provides the differences between the perturbed pressure fields delivered at the end of the calculations associated with either the isolated or the facility-installed NLG configurations; as one can see, compared to the isolated NLG (homogeneous medium) case, when the realistic jet flow is accounted for, the acoustic waves see their patterns modified as they cross the jet shear layers, and then propagate within a region where the medium is at rest. Although they appear to be more prominent upstream the NLG (where the shear layers are denser), these cumulated refraction and (no) convection effects impact almost all the regions located outside the jet, e.g. where the microphones are located. Here, it is worth mentioning that a purely CFD-FWH approach could not be able to provide such assessment, which underlines the interest of employing advanced techniques such as the present CAA-based hybrid method for enhancing the fidelity of noise predictions.

\section{Conclusion}

The present paper recalled the main steps and outcomes of several dedicated research actions that were all conducted within a same wider framework, which ultimate objective is the development of a high fidelity aeroacoustic hybrid approach that can address realistic noise problems. These actions consisted in improving and optimizing an already existing CAA-based hybrid method, which allows weak-coupling CFD (Computational Fluid Dynamics) and CAA (Computational AeroAcoustics) methods and solvers. With that view, the weak-coupling technique was first improved, so that it can handle installed configurations. In a second step, the overall CAA-based hybrid approach was optimized, so that the accuracy of aeroacoustic signals is not questioned by the way the latter are manipulated (i.e. sampled, interpolated, etc.) and/or exploited through CAA means. To this end, various innovative techniques were developed, among which (i) specific criteria for a proper exploitation of aeroacoustic signals, (ii) a novel interpolation method and (ii) a new class of finite differences derivative schemes. All these outcomes were validated and illustrated through various problems of increasing complexity, which were directly inspired by test cases coming from the Category 8 of BANC

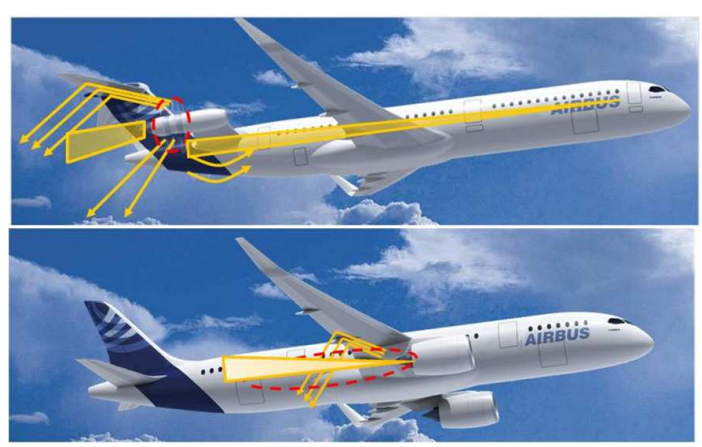

Figure 13. Noise emission and acoustic installation effects of CROR- and HBPR- engines powered aircraft, to be numerically assessed in EC projects JERONIMO and NINHA, respectively (Airbus courtesy).

\footnotetext{
* On that stage, it is worth mentioning that, compared to the signal acquisition time used in the experiment (20 sec), the CFD-CAA simulation time $(0.06 \mathrm{sec})$ was much shorter. In addition to that, because of the transient time needed for the first wave front to reach the probes location, the effective length of the useful signal that was numerically recorded was even shorter (0.04 sec for the far-field microphones). As a consequence, one could expect the spectra analysis of the CFD-CAA outputs to be much less accurate than the one applied to the experimental data. More precisely, while the latter had been Fourier Transform (FT) processed with many averaging blocks and a maximal frequency step (that is, accuracy) of approx. $10 \mathrm{~Hz}$, the former where FT-handled with only a few averages, and a sampling frequency of no less then $100 \mathrm{~Hz}$.
} 
(Benchmark for Airframe Noise Computations). Among other things, this shows how international benchmark efforts such as the ones promoted through the BANC initiative constitute a key means for improving numerical methods in acoustics, whether it is by offering to consolidate further already existing approaches, or by helping in making novel techniques emerge

Thanks to the various actions and outcomes recalled in the present paper, the CAA-based hybrid approach can now cope with stringent constraints that are dictated by realistic aircraft noise problems, without being jeopardized by some of their unavoidable side-effects (acoustic backscattering, signal degradation, etc.). These potentialities shall be illustrated more completely in a very near future, within the framework of two European projects that are devoted to novel aircraft powerplants based on either CROR (Counter Rotating Open Rotor) or HBPR (High By Pass Ratio) engines (see Fig. 13); indeed, for each configuration, the acoustic installation effects resulting from the engines integration will be numerically assessed through weakly coupled CFD-CAA calculations relying on the optimized NRI-based methodology developed in the present framework.

\section{Acknowledgments}

Although the present R\&D works were all funded by ONERA, they took both direct and indirect benefits from several efforts and joint initiatives, among which two internal projects and two international collaborations ${ }^{* \dagger}$; Some works directly benefited from dedicated cooperative tasks that were conducted jointly with researchers from NASA/LaRC (Langley Research Center), which computational means and/or results were used. Other works took benefit from data that were provided by various teams, either coming from other organizations (NASA/LaRC, JAXA) or from Onera (Applied Aerodynamics Department).

Regarding the first part of the present study (see section IV), the first author greatly acknowledges NASA Langley Research Center (LaRC) for having welcomed him and provided him with all logistical means needed, as part of the International Agreement between NASA and ONERA on "Understanding and Predicting the Source of Nose Landing Gear Noise". In particular, he is grateful to Dr. David P. Lockard, Dr. Mehdi Khorrami, and Dr. Meelan Choudhari (all from NASA/LaRC) for their continuous support, help and guidance in the achievement of all related R\&D actions. Regarding the last part of this study (see section VI), authors are grateful to Dr. Saloua Ben Khelil (Onera) for having provided them with CFD datasets associated with BANCIII Category 8.2 benchmark. Regarding all actions, authors thank Dr. Tomoaki Ikeda and Dr. Taro Imamura (JAXA) as well as Dr. Leonard Lopez and Dr. Meelan Choudhari (NASA/LaRC) for the various inputs and insights they provided them with, for the analytical test case (acoustic scattering by a 3D sphere) excerpted from BANC Category 8.1. Finally, authors acknowledge Dr. P. Spalart (Boeing) and Dr. A. Sengissen (Airbus), whose research works and/or informal exchanges encouraged them in conducting the studies presented here.

\section{References}

${ }^{1}$ Ffowcs Williams, J. E. and Hawkings, D. L., "Sound generation by turbulence and surfaces in arbitrary motion," Philosophical Transactions of the Royal Society of London A, Vol. 342, 1969, pp. 264-321.

${ }^{2}$ Lighthill, M.J., "On Sound Generated Aerodynamically. I. General theory / II. Turbulence as a source of sound”, Proc. Roy. Soc. London, Vol. A 211, 1952 / Vol. A 222, 1954.

${ }^{3}$ Ffowcs-Williams, J. E. and Hawkings, D. L., "Sound Generation by Turbulence and Surfaces in Arbitrary Motion", Philosophical Transactions of the Royal Society of London A, Vol. 342, 1969, pp. 264-321.

${ }^{4}$ Tyler, J. M., and Sofrin, T. G., “Axial Flow Compressor Noise Studies”, Transactions of the SAE, Vol. 70, 1962, pp. 309-332.

${ }^{5}$ Redonnet, S., "Simulation de la propagation acoustique en présence d'écoulements quelconques et de structures solides, par résolution numérique des équations d'Euler," PhD Thesis, Université Bordeaux I, 2001.

${ }^{6}$ Redonnet, S., Manoha, E. and Sagaut, P. "Numerical Simulation of Propagation of Small Perturbations interacting with Flows and Solid Bodies," AIAA Paper 2001-2223, 7th CEAS/AIAA Aeroacoustics Conference, Maastricht, The Netherlands, May 2001.

${ }^{7}$ Redonnet, S., Desquesnes, G., Manoha E. and Parzani, C., "Numerical Study of Acoustic Installation Effects with a CAA Method, " AIAA Journal, Vol. 48 n5, May 2010.

${ }^{8}$ Redonnet, S. and Druon, Y., "Computational AeroAcoustics of Realistic Co-Axial Engines," AIAA Journal, Vol 50 n5, May 2012.

${ }^{9}$ Manoha, E., Herrero, C., Sagaut, P. and Redonnet, S., "Numerical Prediction of Airfoil Aerodynamic Noise", AIAA Paper n ${ }^{\circ}$ 2002-2573, June 2002.

${ }^{10}$ Terracol, M., Manoha, E., Herrero, C., Labourasse, E., Redonnet, S. and Sagaut, P., "Hybrid Methods for Airframe Noise Numerical Prediction”, Theoretical and Computational Fluid Dynamics, Vol. 19, n³, July 2005.

${ }^{11}$ Guenanff, R., "Couplage instationnaire Navier-Stokes/Euler pour la génération et le rayonnement des sources de bruit aérodynamique", PhD Thesis, Rennes University, 2004, n³138.

${ }^{12}$ Redonnet, S., "On the Numerical Prediction of Aerodynamic Noise via a Hybrid Approach - Part 1: CFD/CAA Surfacic

\footnotetext{
* International Agreement between NASA and ONERA on "Understanding and Predicting the Source of Nose Landing Gear Noise".

${ }^{\dagger}$ International joint effort for setting up the Benchmark on Airframe Noise Computations (BANC).
} 
Coupling Methodology, Revisited for the Prediction of Installed Airframe Noise Problem", AIAA Paper 2010-3709, June 2010.

${ }^{13}$ Redonnet, S., Lockard, D. P., Khorrami, M. R. and Choudhari, M. M., "CFD-CAA Coupled Calculations of a Tandem Cylinder Configuration to Assess Facility Installation Effects", AIAA Paper 2011-2841, 17th AIAA/CEAS Aeroacoustics Conference, Portland, USA, June 2011.

${ }^{14}$ Cunha, G. and Redonnet, S., "An Innovative Interpolation Technique for Aeroacoustic Hybrid Methods," AIAA Paper 2011-2483, 17th AIAA/CEAS Aeroacoustics Conference, Portland, USA, June 2011.

${ }^{15}$ Tinetti, A. F. and Dunn, M. H., “Acoustic Simulations of an Installed Tandem Cylinder Configuration,” AIAA Paper 2009-3158, 15th AIAA/CEAS AeroAcoustics Conference, Miami, USA, May 2009.

${ }^{16}$ Redonnet, S., Cunha, G., and Ben Khelil, S., "Numerical Simulation of Landing Gear Noise via Weakly Coupled CFDCAA Calculations", AIAA paper 2013-2068, 19th AIAA/CEAS Aeroacoustics Conference, Germany, June 2013.

${ }^{17}$ Lockard D., "Summary of the Tandem Cylinder Solutions from the Benchmark problems for Airframe Noise Computations-I Workshop", AIAA 2011-353, 49th AIAA Aerospace Sciences Meeting, 4-7 January 2011, Orlando, Florida.

${ }^{18}$ Jenkins, L. N., Khorrami, M. R., Choudhari, M. M. and McGinley, C. B., "Characterization of Unsteady Flow Structures Around Tandem Cylinders for Component Interaction Studies in Airframe Noise," AIAA Paper 2005-2812, 11th AIAA/CEAS Aeroacoustics Conference, Monterey, USA, May 23-25, 2005.

${ }^{19}$ Hutcheson, F. V. and Brooks, T. F., "Noise Radiation from Single and Multiple Rod Configurations," AIAA Paper 2006-2629, 12th AIAA/CEAS Aeroacoustics Conference, Cambridge, USA, May 2006.

${ }^{20}$ Lockard, D. P., Khorrami, M. R., Choudhari, M. M., Hutcheson, F. V. and Brooks, T. F., "Tandem Cylinder Noise Prediction," AIAA Paper 2007-3450, 13th AIAA/CEAS Aeroacoustics Conference, Roma, Italy, May 2007.

${ }^{21}$ Cunha G., "Optimization of a Computational Aeroacoustics Methodology Based on the Weak Coupling of Unsteady Aerodynamic and Acoustic Propagation Approaches", PhD Thesis, Toulouse University, 2012

${ }^{22}$ Cunha, G. and Redonnet, S., "On the Signal Degradation Induced by the Interpolation and the Sampling Rate Reduction in Aeroacoustics Hybrid Methods", International Journal for Numerical Methods in Fluids, Vol. 71 (7), February 2013.

${ }^{23}$ Cunha, G. and Redonnet, S., "An Innovative Optimization Methodology for Explicit High-Order Interpolation Schemes", submitted for publication in International Journal for Numerical Methods in Fluids, November 2013.

${ }^{24}$ Cunha, G. and Redonnet, S., "Low-Dispersion High-Order Explicit Finite-Difference Schemes for Computational Aeroacoustics", submitted for publication in International Journal for Numerical Methods in Fluids, October 2013.

${ }^{25}$ S. K. Lele, "Compact finite difference schemes with spectral-like resolution", Journal of Computational Physics 103 (1) (1992) 16-42.

${ }^{26}$ C. K. W. Tam, J. C. Webb, "Dispersion-relation-preserving finite difference schemes for computational acoustics", Journal of Computational Physics 107 (8) (1993) 262-281.

${ }^{27} \mathrm{C}$. Bogey, C. Bailly, "A family of low dispersive and low dissipative explicit schemes for flow and noise computations", Journal of Computational Physics 194 (1) (2004) 194-214.

${ }^{28}$ Manoha E., Bulté J., Caruelle B., "LAGOON : an Experimental Database for the Validation of CFD/CAA Methods for Landing Gear Noise Prediction", AIAA 2008-2816, 14th AIAA/CEAS Aeroacoustics Conference, 5 - 7 May 2008, Vancouver, British Columbia, Canada

${ }^{29}$ Manoha E., Bulté J., Ciobaca V., Caruelle B., "LAGOON : further analysis of aerodynamic experiments and early aeroacoustics results", AIAA 2009-3277, 15th AIAA/CEAS Aeroacoustics Conference, 11 - 13 May 2009, Miami, Florida.

${ }^{30}$ Cambier, L., Heib, S. and Plot, S., "The Onera elsA CFD software: input from research and feedback from industry", Mechanics \& Industry, Vol 14 (3), pp 159-174, January 2013.

${ }^{31}$ Sanders L., Manoha E., Ben Khelil S., François C., "LAGOON : New Mach Landing Gear Noise Computation and further analysis of the CAA process", 18th AIAA/CEAS Aeroacoustics Conference, Colorado Springs, USA, June 2012.

${ }^{32}$ Prieur J. and Rahier G., "Aeroacoustic integral methods, formulation and efficient numerical implementation", Aerospace Science and Technology, Vol. 5 (7), October 2001, pp. 457-468. 